\title{
A Hybrid Experimental/Theoretical Scattering Model for Dense Random Media
}

\author{
John R. Kendra, Member, IEEE, and Kamal Sarabandi, Senior Member, IEEE
}

\begin{abstract}
The subject of scattering of electromagnetic waves by dense media has been one of intense interest in recent years. The present paper describes polarimetric backscatter measurements made at $K u$-band on layers of a dense medium under very carefully controlled circumstances. The experiments have a dual purpose: 1) to evaluate the degree to which the experimental observations are predicted by theoretical, particle-based, random media models and 2) to test a proposed hybrid model by which the scattering and extinction properties of a dense medium are characterized experimentally, allowing future modeling of the polarimetric response for any arbitrary configuration of the medium. The hybrid model assumes that first-order vector radiative transfer (RT) is a suitable theoretical structure, providing that the extinction and phase matrix components are appropriately specified; the specification is accomplished through an inversion algorithm involving polarimetric backscatter measurements. The major conclusions of the study are the following:

1) hybrid model is an adequate description of the dense medium scattering behavior;

2) conventional RT appears to give a reasonable estimate of the observed radar response, but dense medium $R T$ gives a very low estimate;

3) phase function of the effective volume scattering element of the medium, obtained via the hybrid model, suggests a larger effective scatterer than the physical ones.
\end{abstract}

Index Terms - Random media, scattering and propagation, scattering model.

\section{INTRODUCTION}

$\mathbf{E}$ VIDENCE has recently been presented [1], [2] which suggests that existing particle-based theories are inadequate for modeling very dense random media. This raises the very fundamental question of what recourse exists for simulating the effects of a dense medium, for example, for the case of a snow layer over sea ice or on a forest floor. The ability to generate realistic predictions is an essential prerequisite to any scheme for retrieving physical characteristics about a target from remotely sensed data.

If the behavior of densely packed discrete particles is difficult to model, it is evident that the issue is only exacerbated when dealing with materials of a very complex physical character, for example, the amorphous interconnected

Manuscript received October 3, 1995; revised July 7, 1997. This work was supported in part by ONR under Contract N00014-95-1-0736 and JPL under Contract 958749.

J. R. Kendra is with the $\mathrm{C}^{3}$ I Group, Raytheon Systems Company, Dallas, TX 75266 USA.

K. Sarabandi is with the Radiation Laboratory, Department of Electrical Engineering and Computer Science, The University of Michigan, Ann Arbor, MI 48109-2122 USA (e-mail: saraband@eecs.umich.edu).

Publisher Item Identifier S 0196-2892(99)00090-X. form that snowpacks can often-or even usually—take. There are of course theoretical techniques that could be applied to such cases; these are field-based techniques in which the medium is described as a fluctuating dielectric constant. To obtain a solution, the Born approximation or the distorted Born approximation is usually applied. In practice, however, although some studies have investigated this technique [3], the particular material characteristic that is required as an input, the correlation function of the medium, is exceedingly difficult to obtain. The standard technique for its measurement is the "thin sections" technique. In this technique [3], [4], a supercooled liquid is allowed to fill the pore spaces of a snow sample. After freezing the samples, they are shaved on a microtome, polished, treated with a contrast enhancer, and digitized. Not only is this a very arduous process, but it has been recently shown [5] that the correlation function must be known with high accuracy, including its tail region, to obtain accurate prediction of scattering; that is, it is not sufficient to know that the function approximates, say, a Gaussian or exponential function. In addition to these obstacles, it is also true that the distorted Born approximation does not account for multiple scattering of the incoherent wave [6].

Apart from the theoretical approaches addressed above, purely empirical approaches may be considered; these, however, have the obvious limitation that the entire parameter space of the target cannot be sufficiently well known to allow estimation of more specific target properties.

To circumvent the difficulties associated with the abovementioned techniques and to offer some means by which realistic modeling of dense media might be accomplished, a new hybrid experimental/theoretical modeling scheme is introduced in this paper. In the sections that follow, we will describe the hybrid model and address its validity with actual polarimetric backscatter measurements on a dense medium. The measured results will also be compared with certain discrete particle-based random media theories.

\section{Hybrid Model CONCEPT}

The hybrid model involves two major assumptions. The first is that radiative transfer (RT) theory is applicable. That is, that the flow of electromagnetic energy through any dense medium obeys the fundamental equation of transfer

$$
\frac{d \mathbf{I}(\hat{\mathbf{s}}, \mathbf{r})}{d s}=-\kappa_{\boldsymbol{e}}(\hat{\mathbf{s}}) \mathbf{I}(\hat{\mathbf{s}}, \mathbf{r})+\int_{\theta=0}^{\pi} \int_{\phi=0}^{2 \pi} \mathcal{P}\left(\hat{\mathbf{s}}, \hat{\mathbf{s}}^{\prime}\right) \mathbf{I}\left(\hat{\mathbf{s}}^{\prime}, \mathbf{r}\right) d \Omega^{\prime}
$$


where $\mathbf{I}$ is the $4 \times 1$ Stokes vector, $\boldsymbol{\kappa}_{\boldsymbol{e}}$ is the $4 \times 4$ extinction matrix, and $\mathcal{P}$ is the $4 \times 4$ phase matrix. The phase and extinction matrices for a medium may be formulated in terms of the characteristics of a single particle if the medium consists of uniform particles or a weighted average of the individual characteristics of various particles if the medium is heterogeneous [7]. This type of formulation, known as conventional radiative transfer (CRT), contains two simplifying assumptions: 1) that all particles are in the farfield of one another and 2) that the particle positions are completely uncorrelated with one another, thereby eliminating any coherence effects.

It has also been shown [8] that for Rayleigh particles, a rigorous field-based approach to scattering in a dense medium, where particle correlations are considered and exact wave transformations from one particle to another are employed, yields a solution that may be couched in exactly the same form as (1). It was found from this analysis that the phase function is the Rayleigh phase function, similar to CRT. However, the scale of the phase function, which is provided by the scattering albedo, and the extinction differ from CRT. Thus, the assumption that such an equation describes the mechanism is reasonable, although it has not been shown to hold for the case of a rigorous field-based analysis of non-Rayleigh particles [9].

We have already mentioned above studies in which evidence is provided suggesting that the available techniques by which $\boldsymbol{\kappa}_{e}$ and $\mathcal{P}$ are computed do not appear to be adequate. In addition, for complex or amorphous materials, in which a "particle" cannot be unambiguously identified, it is not possible to formulate these quantities. Therefore, in the present hybrid model, no attempt is made to specify them based on the physical characteristics of the medium. Instead, a direct measurement process will be carried out, effectively interrogating the medium, and from these measurements, an inversion process will be used to retrieve $\kappa_{e}$ and $\mathcal{P}$.

In order to make this approach tractable, a second major assumption is required, which is that, not only does RT apply, but that the first-order evaluation of it is sufficient. The firstorder solution to the RT equation for the case of an isotropic layer with smooth surfaces above and below has been derived in [7]. For

$$
\mathbf{I}^{s}\left(\mu_{o}\right)=\mathcal{L}_{m}\left(\mu_{o}\right) \mathbf{I}^{i}\left(-\mu_{o}\right)
$$

where $\mathcal{L}_{m}$ is transformation matrix related to the Mueller matrix $\mathcal{M}$ [7] by

$$
\mathcal{L}_{m}=\frac{1}{A \cos \theta_{i}} \mathcal{M}
$$

where $A$ is the illuminated area and $\theta_{i}$ is the angle of incidence, we have

$$
\begin{aligned}
& \mathcal{L}_{m}\left(\mu_{o}\right)=\frac{1}{\mu_{o}^{\prime}} \mathcal{T}_{21}\left[\gamma \mathcal{R}_{23} \mathcal{P}_{b s} \mathcal{R}_{23}+\frac{d}{L} \mathcal{R}_{23} \mathcal{P}_{b i}\right. \\
& \left.+\frac{d}{L} \mathcal{P}_{b i} \mathcal{R}_{23}+\gamma \mathcal{P}_{b s}\right] \mathcal{T}_{12}
\end{aligned}
$$

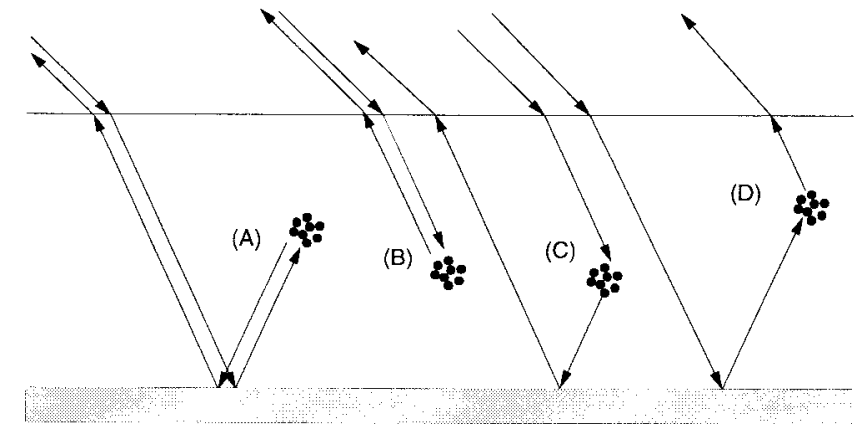

Fig. 1. First-order volume scattering mechanisms in a layer of scatterers.

where

$$
\begin{aligned}
& \gamma=\frac{1-\exp \left(-2 \kappa_{e} d / \mu_{o}^{\prime}\right)}{\left.2 \kappa_{e} / \mu_{o}^{\prime}\right)} \\
& L=\exp \left(2 \kappa_{e} d / \mu_{o}^{\prime}\right)
\end{aligned}
$$

where $\kappa_{e}$ is the extinction (a scalar quantity) and $\mathcal{P}_{b s}$ and $\mathcal{P}_{b i}$ are the backscatter and bistatic components of the phase matrix, respectively; $\mathcal{R}_{p q}$ and $\mathcal{T}_{p q}$ are the respective Fresnel reflectivity and transmissivity matrices associated with intensity propagating in medium $p$ toward medium $q$, and $\mu_{o}^{\prime}$ is the cosine of the refracted angle in the medium.

The four scattering mechanisms described by (4) are shown in Fig. 1. The scattering event depicted in terms (A) and (B) are associated with the backscatter component of the phase function $\mathcal{P}_{b s}$; terms $(\mathrm{C})$ and $(\mathrm{D})$ are associated with the bistatic component of the phase function $\mathcal{P}_{b i}$. In the figure, we have intentionally represented the scattering elements as clusters to underscore the point that, in this treatment, we are considering effective particles, which may comprise correlated groups of individual physical particles and/or multiple scattering effects.

\section{Rayleigh Model for Phase AND EXTINCTION MATRICES}

In this section, we examine, as an aid to inversion of the hybrid model, the essential character of the extinction matrix $\kappa_{e},{ }^{1}$ and the two components of the phase matrix $\mathcal{P}_{b s}$ and $\mathcal{P}_{b i}$, for the special case of scattering by a layer of Rayleigh particles. This scenario corresponds to the isotropic case mentioned above, in which $\boldsymbol{\kappa}_{e}$ and $\mathcal{P}_{b s}$ are constant matrices, but $\mathcal{P}_{b i}$ is a function of angle.

The analysis is motivated by the intuition that the number of measurements required and the complexity of the ensuing inversion operation could be reduced through knowledge of the general form of the unknown matrices; that is, knowledge of which elements are nonzero, which are independent, and the nature of the dependencies between elements.

As a starting point, we consider the effective particles of the layer (as depicted in Fig. 1) to be Rayleigh particles. As we will show in the following sections, this will allow us to derive symbolically the specific mathematical structure of the quantities of interest-the extinction matrix and certain components of the phase matrix consistent with a first-order

\footnotetext{
${ }^{1}$ The analysis begins with the most general case of a matrix extinction, as derived in [10]
} 
approach-in terms of the elements of the polarizability tensor of the particles. This derivation follows closely that found in [11], except that in that case only symmetric particles were considered, whereas, in the present case, no assumptions are made about the particle's symmetry properties.

\section{A. Rayleigh Theory: Scattering Matrix Element Representation}

For particles with small dimensions relative to the wavelength, scattering may be described in terms of a polarizability tensor. A scattered electric field vector is given by [12]

$$
\mathbf{E}^{s}=\frac{-\omega^{2} \mu}{4 \pi r} e^{i k_{o} r} \hat{\mathbf{k}}_{s} \times \hat{\mathbf{k}}_{s} \times \mathbf{p}
$$

where $\hat{\mathbf{k}}_{s}$ is the unit vector in the direction of propagation of the scattered wave and $\mathbf{p}$ is the induced dipole moment determined from the solution of the Laplace equation. If the induced dipole moments $\left(\mathbf{p}_{x}, \mathbf{p}_{y}, \mathbf{p}_{z}\right)$ are derived for $(\hat{\mathbf{x}}, \hat{\mathbf{y}}, \hat{\mathbf{z}})$ polarized incident fields, the scattered field may be expressed as

$$
\mathbf{E}^{s}=\frac{-\omega^{2} \mu}{4 \pi r} e^{i k_{o} r} \hat{\mathbf{k}}_{s} \times \hat{\mathbf{k}}_{s} \times\left(\mathcal{D} \cdot \mathbf{E}^{i}\right)
$$

where $\mathcal{D}$ is the $3 \times 3$ polarizability tensor, having as its columns the vectors $\mathbf{p}_{x}, \mathbf{p}_{y}$, and $\mathbf{p}_{z}$, and $\mathbf{E}^{i}$ is the incident field vector $\left[E_{x}, E_{y}, E_{z}\right]^{T}$. The polarizability tensor $\mathcal{D}$ is a function of the geometry and dielectric constant of the Rayleigh particle. In general, $\mathcal{D}$ will be a function of the orientation of the particle.

Since

$$
\mathbf{E}^{s}=\frac{e^{i k_{o} r}}{r} \mathbf{S} \cdot \mathbf{E}^{i}
$$

where

$$
\mathbf{S}=\left(\begin{array}{ll}
S_{v v} & S_{v h} \\
S_{h v} & S_{h h}
\end{array}\right)
$$

it can be shown [13] that the scattering matrix elements corresponding to the scattered polarization $\hat{\mathbf{p}}_{s}$ and the incident polarization $\hat{\mathbf{q}}_{i}$ such that $\hat{\mathbf{p}}_{s} \in\left\{\hat{\mathbf{v}}_{s}, \hat{\mathbf{h}}_{s}\right\}$ and $\hat{\mathbf{q}}_{i} \in\left\{\hat{\mathbf{v}}_{i}, \hat{\mathbf{h}}_{i}\right\}$ are given by

$$
S_{p q}=\frac{k_{o}^{2}}{4 \pi} \hat{\mathbf{p}}_{s} \cdot\left(\mathcal{D} \cdot \hat{\mathbf{q}}_{i}\right) .
$$

\section{B. Derivation of Extinction and Phase Matrix Elements}

The extinction and phase matrix elements are expressed in terms of the ensemble-averaged quantities $\left\langle S_{p q}\right\rangle$ [10] and $\left\langle S_{p q} S_{m n}^{*}\right\rangle$ [7], respectively. In this section, we present the derivation of the forms of these elements in terms of the elements of the polarizability tensor in global coordinates.

1) Extinction Matrix Elements: We consider first terms of the form $\left\langle S_{p q}\right\rangle$. From (11)

$$
\left\langle S_{p q}\right\rangle=\frac{k_{o}^{2}}{4 \pi}\left\langle\hat{\mathbf{p}}_{s} \cdot\left(\mathcal{D} \cdot \hat{\mathbf{q}}_{i}\right)\right\rangle .
$$

Since the polarization vectors themselves are not a function of the particle orientation, the averaging process is performed only on $\mathcal{D}$ itself

$$
\left\langle S_{p q}\right\rangle=\frac{k_{o}^{2}}{4 \pi} \hat{\mathbf{p}}_{s} \cdot\left(\langle\mathcal{D}\rangle \cdot \hat{\mathbf{q}}_{i}\right) .
$$

The form of $\mathcal{D}$ in local coordinates is transformed from that in global coordinates $\left(\mathcal{D}^{\prime}\right)$ through the application of transformation matrices involving the Eulerian rotation angles $\alpha, \beta$, and $\gamma$ (see, for example, [14, pp. 158-160])

$$
\mathcal{D}=\mathcal{A}^{T} \cdot \mathcal{D}^{\prime} \cdot \mathcal{A}
$$

where the elements of the $3 \times 3$ transformation matrix $\mathcal{A}$ are trigonometric functions of $\alpha, \beta$, and $\gamma$, e.g.,

$$
\begin{aligned}
& a_{11}=\cos \gamma \cos \beta \cos \alpha-\sin \gamma \sin \alpha \\
& a_{12}=\cos \gamma \cos \beta \sin \alpha+\sin \gamma \cos \alpha \\
& a_{12}=-\cos \gamma \sin \beta
\end{aligned}
$$

Therefore, for each element of $\mathcal{D}$, the polarizability tensor in local coordinates is

$$
d_{i j}=\left(\mathcal{A}^{T} \cdot \mathcal{D}^{\prime} \cdot \mathcal{A}\right)_{i j}=\sum_{m, n=1}^{3}\left(a_{m i} a_{n j}\right) d_{m n}^{\prime} .
$$

It is seen from (16) that the symmetry property of $\mathcal{D}$ is preserved across a coordinate transformation.

For extinction, the quantity $\left\langle S_{p q}\right\rangle$ is computed in the forward direction only. Because of the assumption of classical RT that the positions of particles are uncorrelated, $\left\langle S_{p q}\right\rangle$ will be zero for every direction but forward scattering.

Since we have assumed there is no orientation dependence, we can assign the simplest possible forms to the vertical and horizontal polarization vectors, incident and scattered, and compute $\left\langle S_{p q}\right\rangle$ from (13). Considering an incident direction along the positive $x$-axis, using the forward scattering convention (FSA) [7], we have,

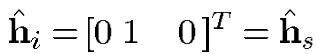

$$
\begin{aligned}
& \hat{\mathbf{v}}_{i}=\left[\begin{array}{lll}
0 & 0 & -1
\end{array}\right]^{T}=\hat{\mathbf{v}}_{s} \text {. }
\end{aligned}
$$

Ignoring the factor $k_{o}^{2} / 4 \pi$ and applying (13)

$$
\begin{aligned}
& \left\langle S_{v v}\right\rangle=\left\langle d_{33}^{\prime}\right\rangle \\
& \left\langle S_{h h}\right\rangle=\left\langle d_{22}^{\prime}\right\rangle \\
& \left\langle S_{h v}\right\rangle=-\left\langle d_{23}^{\prime}\right\rangle \\
& \left\langle S_{v h}\right\rangle=-\left\langle d_{32}^{\prime}\right\rangle=-\left\langle d_{23}^{\prime}\right\rangle .
\end{aligned}
$$

From (16)

$$
\begin{aligned}
\left\langle d_{i j}\right\rangle= & \int_{\alpha=0}^{2 \pi} \int_{\beta=0}^{\pi} \int_{\gamma=0}^{2 \pi} \sum_{m, n=1}^{3} \\
& \times\left(a_{m i} a_{n j}\right) d_{m n}^{\prime} p(\alpha) p(\beta) p(\gamma) d \alpha d \beta d \gamma
\end{aligned}
$$

where, for completely uniform orientation, the respective probability density functions are

$$
\begin{aligned}
& p(\alpha)=\frac{1}{2 \pi} \\
& p(\beta)=\frac{\sin \beta}{2} \\
& p(\gamma)=\frac{1}{2 \pi}
\end{aligned}
$$


over the limits indicated on the integrals in (19). These integrals may be evaluated without difficulty, and the results are

$$
\begin{aligned}
& \left\langle d_{22}\right\rangle=\frac{1}{3}\left(d_{11}^{\prime}+d_{22}^{\prime}+d_{33}^{\prime}\right) \\
& \left\langle d_{33}\right\rangle=\left\langle d_{22}\right\rangle \\
& \left\langle d_{23}\right\rangle=0 .
\end{aligned}
$$

Thus, for the scenario under consideration, the extinction is found to be a scalar [as was already assumed in (4)] and-when the result above is used in the definition [7] for the extinction elements-we obtain the following expression:

$$
\kappa_{e}=-2 \operatorname{Re}\left[\frac{-2 j \pi n_{\circ}}{3 k_{\circ}}\left(d_{11}^{\prime}+d_{22}^{\prime}+d_{33}^{\prime}\right)\right]
$$

where $n_{\circ}$ is the particle-number density.

2) Phase Matrix Elements: The derivation of the phase matrix elements involves evaluation of quantities of the form $\left\langle S_{p q} S_{m n}^{*}\right\rangle$. Referring back to (11), we have

$$
\left\langle S_{p q} S_{m n}^{*}\right\rangle=\frac{k_{o}^{4}}{(4 \pi)^{2}}\left\langle\left(\hat{\mathbf{p}}_{s} \cdot \mathcal{D} \cdot \hat{\mathbf{q}}_{i}\right)\left(\hat{\mathbf{m}}_{s} \cdot \mathcal{D}^{*} \cdot \hat{\mathbf{n}}_{i}\right)\right\rangle .
$$

The derivation is similar to that performed for the extinction matrix elements, but it is considerably more involved. The problem of evaluating (23) essentially reduces to evaluating the spatial ensemble average of the Kronecker tensor product

$$
\left\langle\mathcal{D} \otimes \mathcal{D}^{*}\right\rangle
$$

where $\mathcal{D}$ is the polarizability tensor in local coordinates whose elements are given by (16). The Kronecker tensor product, however, inflates the formulation greatly since each element $d_{i j}$ in $\mathcal{D}$ is composed of approximately 12 terms (since some of the elements in the transformation matrix $\mathcal{A}$ have two terms), each element in $\left\langle\mathcal{D} \otimes \mathcal{D}^{*}\right\rangle$, a $9 \times 9$ matrix, has on the order of 144 terms, each requiring analytical integration with respect to the three Eulerian rotation angles $\alpha, \beta$, and $\gamma$. The details of this derivation are given in [13]. The result of the analysis is the extremely simple form in (25) and (26), shown at the bottom of the page, where

$$
\begin{aligned}
P_{1} & =\frac{1}{5} A+\frac{4}{15} B+\frac{2}{15} C \\
P_{2} & =\frac{1}{15} A-\frac{2}{15} B+\frac{4}{15} C \\
A & =\left|d_{11}^{\prime}\right|^{2}+\left|d_{22}^{\prime}\right|^{2}+\left|d_{33}^{\prime}\right|^{2} \\
B & =\left|d_{12}^{\prime}\right|^{2}+\left|d_{13}^{\prime}\right|^{2}+\left|d_{23}^{\prime}\right|^{2} \\
C & =\operatorname{Re}\left(d_{11}^{\prime} d_{22}^{*}+d_{11}^{\prime} d_{33}^{*}+d_{22}^{\prime} d_{33}^{*}\right) .
\end{aligned}
$$

Thus, an analytical evaluation of the theoretical problem of backscattering from a layer of general Rayleigh particles reveals that scattering and extinction within the layer is described by just three parameters: a scalar extinction $\kappa_{e}$ and the two scalar parameters $P_{1}$ and $P_{2}$. This extremely simple form can potentially be exploited in the inversion process associated with the hybrid model concept.

This completes the theoretical framework of the hybrid model concept. The remaining elements are an experimental process and a numerical inversion algorithm. These are described in the following sections.

\section{EXPERIMENTAL PROCESS}

An experimental effort was carried out to test the validity of the hybrid model concept. A photograph of the experimental setup is shown in Fig. 2. The experimental process was straightforward. Polarimetric backscatter measurements of a dense material were made with a $K u$-band network-analyzerbased radar. Details of the specific material examined are given in Section VI. The material examined was contained inside a large 1.8- $\times 1.8-\mathrm{m}$ wooden "sandbox." The sandbox itself was mounted on top of a turntable to allow for independent spatial measurements. The sides of the sandbox were two tiered with the top tier removable so that it could have a maximum depth of either 23 or $43 \mathrm{~cm}$. This was useful to prevent an excessive amount of "shadowing" of the surface of the layer for shallow depths. The radar was mounted on a manually adjustable, telescoping mechanical lift. The radar mount was such that manual adjustment of the incidence angle could be accomplished by simply tilting it and locking it into place. The range from the aperture of the radar antenna to the layer surface, along the boresight direction of the antenna was always $3 \mathrm{~m}$. This distance corresponds to approximately $D^{2} / \lambda$, where $D$ is the dimension of the square aperture of the antenna, which was sufficiently small to allow a full range of incidence angles to be observed in an indoor setting.

As is described fully in [13], the radar consists of upconversion, downconversion, and polarization selection circuitry attached to a square horn antenna via an orthomode transducer. A coherent IF signal at $1-3 \mathrm{GHz}$ is provided by the network analyzer and mixed with a local oscillator at the radar box to produce a 15-17-GHz RF. The design and operation of the radar follows closely that of the LCX POLARSCAT system

$$
\begin{aligned}
\mathcal{P}_{b s} & =\left[\begin{array}{cccc}
P_{1} & \frac{1}{2}\left(P_{1}-P_{2}\right) & 0 & 0 \\
\frac{1}{2}\left(P_{1}-P_{2}\right) & P_{1} & 0 & 0 \\
0 & 0 & -\frac{1}{2}\left(P_{1}+P_{2}\right) & 0 \\
0 & 0 & 0 & \frac{1}{2}\left(P_{1}-3 P_{2}\right)
\end{array}\right] \\
\mathcal{P}_{b i} & =\left[\begin{array}{cccc}
\frac{1}{2} P_{1}\left(1+\cos ^{2}(2 \theta)\right)-\frac{1}{2} P_{2} \sin ^{2}(2 \theta) & \frac{1}{2}\left(P_{1}-P_{2}\right) & 0 & 0 \\
\frac{1}{2}\left(P_{1}-P_{2}\right) & P_{1} & 0 & 0 \\
0 & 0 & \frac{1}{2} \cos 2 \theta\left(P_{1}+P_{2}\right) & 0 \\
0 & 0 & 0 & -\frac{1}{2} \cos 2 \theta\left(P_{1}-3 P_{2}\right)
\end{array}\right]
\end{aligned}
$$




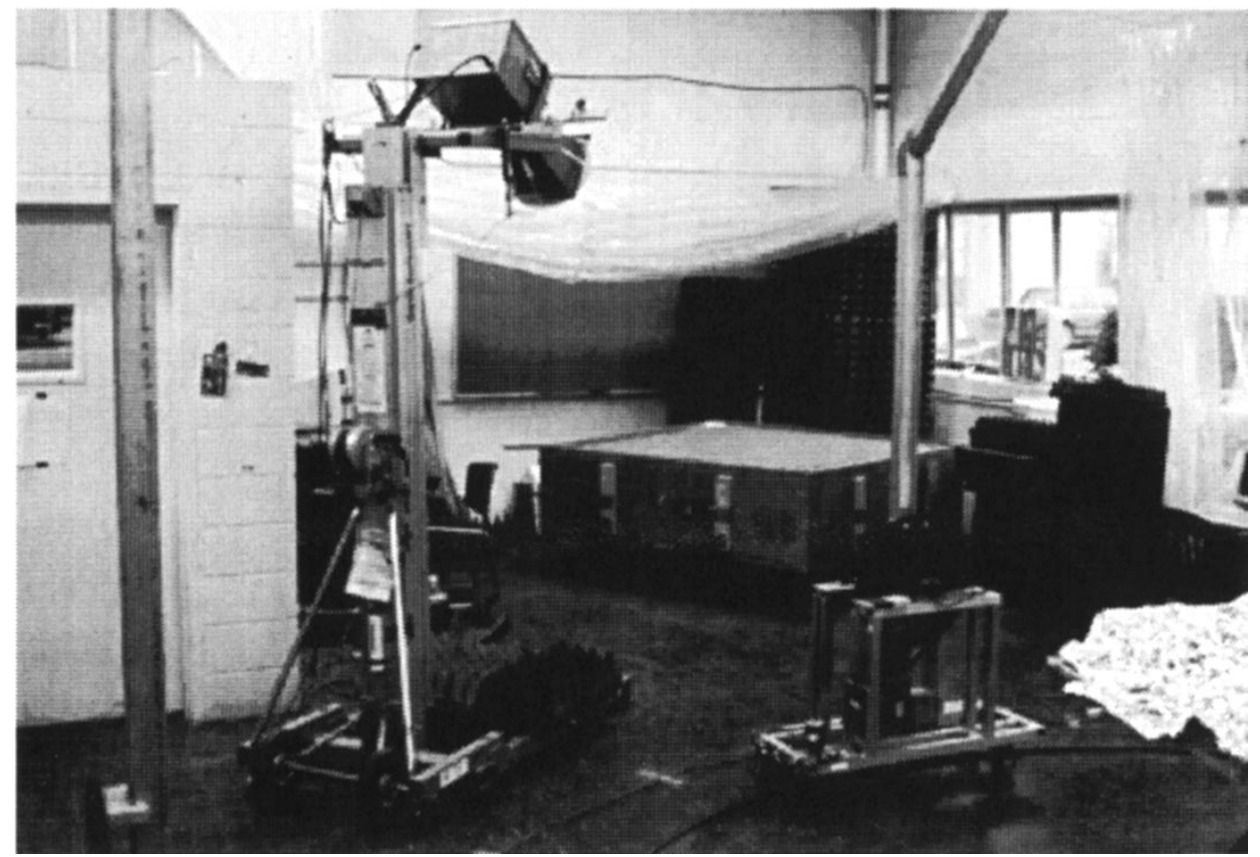

Fig. 2. Photograph of experimental setup. Radar mounted on a mechanical lift overlooking a sandbox filled with the test material. (Additional equipment is part of an unrelated bistatic facility.)

described in [15]. A compact geometry for the antenna is enabled through the use of a dielectric lens to correct the phase error at the aperture. The final beamwidth for the lenscorrected antenna (one-way, HPBW) was $5.3^{\circ}$ for both E- and H-plane.

Two target parameters were varied in the experimental process for each of the materials examined: 1) layer depth and 2) the nature of the underlying "halfspace." The layer depth was varied between $2 \mathrm{~cm}$ and about $40 \mathrm{~cm}$. For the underlying surface, either an aluminum sheet $\left(\tilde{\epsilon}=1-j 3.97 \times 10^{7}\right.$ at $\left.16 \mathrm{GHz}\right)$ or a flat absorber was used. The flat absorber was composed of $60-\times 60-\mathrm{cm}$ slabs of $5-\mathrm{cm}$ thick flat absorber. These slabs were then arranged to cover the bottom of the sandbox. The dielectric constant of the absorber $(\tilde{\epsilon}=1.64-j 0.15)$ was measured by comparing the nadir-viewing reflectivity relative to the conducting sheet. The imaginary part was estimated (a lower limit) by considering the minimum amount that the reflection from a conducting sheet under the absorber had been attenuated.

Both the radar operation and the movement of the turntable were under computer control. To reduce the variance in the estimate of the mean backscatter, independent measurements were realized by rotating the sandbox. It was found that the signal was sufficiently decorrelated for a rotation of $5^{\circ}$. Thus, for each target scenario examined (incidence angle, depth, underlying dielectric), 72 independent spatial samples were collected, one every $5^{\circ}$ for one complete revolution of the sandbox. Additional averaging was obtained using frequency averaging, normally using the responses from 21 frequency points equally spaced throughout the $2-\mathrm{GHz}$ bandwidth. The gating capability of the network analyzer was used to isolate the backscatter response of the layer. The footprint of the antenna at 3 meters-even at $60^{\circ}$, the highest incidence angle examined—was sufficiently small $(<0.56 \mathrm{~m})$ so that scattering from the wooden sides of the sandbox constituted a negligible interference.

Since the primary goal of this investigation is to study volume scattering, great care was taken to make the surface of the layers as smooth as possible to minimize surface backscatter. The smoothing technique employed a sled whose skids rode along two opposite sides of the sandbox and from which was suspended, spanning the entire width of the sandbox, a metal blade. The metal blade was lowered to the appropriate depth and leveled. The sled was then pulled across the box multiple times. After each pass, excess material was removed manually or additional material added in which the level was perceived to be low. In this fashion, an optimally smooth and reproducible surface, with roughness dictated mainly by the particle size of the test material, was achieved. The roughness of the surface was accurately characterized by measurement with a laser profiler.

For calibration of the radar, the isolated antenna calibration technique (IACT) was used [16], which requires the measurement of a sphere (a 6-in sphere, in this case) and an arbitrary depolarizing target (we used a length of wire oriented at roughly $45^{\circ}$ ). This technique, as the name implies, requires a radar system with excellent polarization isolation. The $K u$-band system has polarization isolation on the order of $30 \mathrm{~dB}$.

\section{INVERSION ALGORITHM}

In general, the inversion process consists of two steps, as follows.

1) Assume some form of the unknown matrices $\kappa_{\boldsymbol{e}}, \mathcal{P}_{b s}$, and $\mathcal{P}_{b i}$. A logical starting point is for example, an isotropic model using the three parameters $\kappa_{e}, \mathbf{P}_{1}$, and $\mathbf{P}_{2}$ from the Rayleigh derivation above. 
2) Use an optimization algorithm to minimize the leastsquares function given by

$$
\sum_{i=1}^{N_{\theta}} \sum_{j=1}^{N_{d}} \sum_{k=1}^{N_{c}} \sum_{l=1}^{4} \sum_{m=1}^{4}\left\{\frac{\left[\mathcal{M}_{i, j, k}\right]_{l, m}-\left[\overline{\mathcal{M}}_{i, j, k}\right]_{l, m}}{\left[\mathcal{M}_{i, j, k}\right]_{l, m}}\right\}_{(27)}^{1 / 2}
$$

where $\mathcal{M}$ and $\overline{\mathcal{M}}$ are the measured and predicted Mueller matrices, respectively, corresponding to all of the different experimental variables-incidence angle, depth, and dielectric of underlying surface—indexed up to $N_{\theta}, N_{d}$, and $N_{\epsilon}$.

The formulation of $\overline{\mathcal{M}}$ is nonlinear, even for the simple case of a scalar extinction, due to the presence of the exponential functions of the extinction. Initially, a conjugate gradient procedure was attempted but was found to be unsatisfactory because of the absence of a means for enforcing upper and lower limits on the parameters being estimated. It is, for example, a physical requirement that the parameters corresponding to $\left\langle S_{v v} S_{v v}^{*}\right\rangle,\left\langle S_{h h} S_{h h}^{*}\right\rangle$, and $\left\langle S_{v h} S_{v h}^{*}\right\rangle$ be strictly positive. We eventually settled upon a very robust algorithm written by the Institute for Systems Research, University of Maryland, College Park [17], called FSQPD (Fortran sequential quadratic programming double precision). The algorithm allows upper and lower bounds to be set for the parameters and provides for the imposition of linear and nonlinear constraints, though none were used in this case.

\section{MATERIAL EXAMINED}

The most interesting application of the hybrid modeling concept would be snow. However, in this development stage, the handling requirements of snow for the purpose of a (possible lengthy) set of controlled experiments are too prohibitive. In this initial development stage, therefore, we opted to use a "stable" material, for which material characteristics are static and unchanging throughout the layer.

The dense medium was a sized silica gravel, henceforth referred to as " $8-12$ gravel," based on its being produced in a sieving process as the material retained on standard sieves $8-12$. Some characteristics of this material are given in Fig. 3, which shows the particle size distribution obtained from sieve analyses; also given in the figure are some pertinent physical and electrical properties of the material. The material has a high volume fraction that indicates a maximum packing density of particles. The particle-size distribution of the 8-12 gravel has been rendered as a probability density function (pdf) to facilitate, as will be discussed below, its comparison with theoretical scattering predictions.

The effective dielectric constant of the 8-12 material was measured in two different ways: 1) a coaxial waveguide complex reflection coefficient technique at $9.5 \mathrm{GHz}$ and 2) using the snow probe [18] at about $1.5 \mathrm{GHz}$. While the $9.5-\mathrm{GHz}$ result would seem to be most pertinent to the present effort, which is conducted at $15-17 \mathrm{GHz}$, there are questions about the accuracy of the estimate of the imaginary part of the dielectric constant, which is frequency dependent, as measured by the waveguide technique. The issue of determining $\tilde{\epsilon}$ for the materials is addressed further in Section IX. For now, we

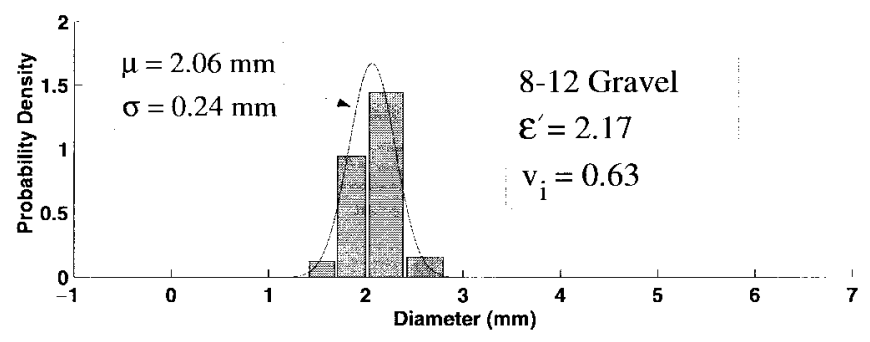

Fig. 3. Characteristics of the test material, $8-12$ gravel. $\left(\nu_{i}\right.$ denotes volume fraction.)

TABLE I

Material Characteristic OF 8-12 Gravel

\begin{tabular}{l|l||l|l|}
\hline \hline \multicolumn{2}{c||}{ Typical Physical Properties } & \multicolumn{2}{c}{ Typical Chemical Analysis } \\
\hline Fusion Point & $31355^{\circ} \mathrm{F}$ & Silicon dioxide & $98.20 \%$ \\
\hline Hardness & 7 Mhos scale & Aluminum oxide & $0.72 \%$ \\
\hline Grain Shape & Spherical & Calcium oxide & $0.56 \%$ \\
\hline Specific gravity & $2.65 \mathrm{~g} / \mathrm{cm}^{3}$ & Irom oxide & $0.07 \%$ \\
\hline Bulk density & $\approx 1.57 \mathrm{~g} / \mathrm{cm}^{3}$ & Magnesium oxide & $0.13 \%$ \\
\hline pHI & 6.87 .2 & Manganese oxide & $0.03 \%$ \\
\hline Sphericity (Krumbein) & $0.6-0.7$ & Potassium oxide & $0.10 \%$ \\
\hline Roundness (Krumbein) & $0.6-0.7$ & Sodium oxide & $0.03 \%$ \\
\hline & & Titanium oxide & $0.07 \%$ \\
\hline & & Loss on ignition & $0.09 \%$ \\
\hline
\end{tabular}

show in Fig. 3 only the real part of the dielectric constants of the materials, $\epsilon^{\prime}$, which is essentially the same at both of the frequencies at which it was measured.

A detailed description of the 8-12 gravel, provided by the supplier (AGSCO Corp., Wheeling, IL) is given in Table I. As shown in Fig. 3, this material has a relatively narrow particlesize distribution, which corresponds to a Gaussian pdf having mean diameter $\mu=2.062 \mathrm{~mm}$ and variance $\sigma^{2}=0.056 \mathrm{~mm}$. The particles themselves have a high degree of sphericity. Such factors make it an excellent candidate for testing against theoretical RT models. At $K u$-band $(\lambda=1.875 \mathrm{~cm}$ at midband), the associated size parameter $k a$, where $k$ is the wavenumber in the particle and $a$ is its radius, is just beyond the Rayleigh limit, for which the criterion has been given as $k a<0.5$ [19] (present case: $k a=0.509$ ). Although the DMRT model [6] is intended specifically for Rayleigh particles, applications have been demonstrated by its authors for cases having $k a$ as high as unit 1 [20]; thus, a meaningful comparison should be permissible with the 8-12 material. One obstacle to theoretical comparisons is the problem of precisely ascertaining the precise complex dielectric constant of the particles themselves at $K u$-band. The issue of a theoretical comparison with measurements is taken up in Section IX.

\section{SURFACE CONTRIBUtions}

In Section IV, a means by which the top surface of the layer was made as smooth as possible was presented. In order to carefully analyze the scattering behavior of the volume, the effect of the surface must be either 1) very well understood or 2) negligible. Since the physics of rough surface scattering is in itself a very challenging problem, and doubly so when integrated with volume scatter, we have attempted to achieve option 2). 


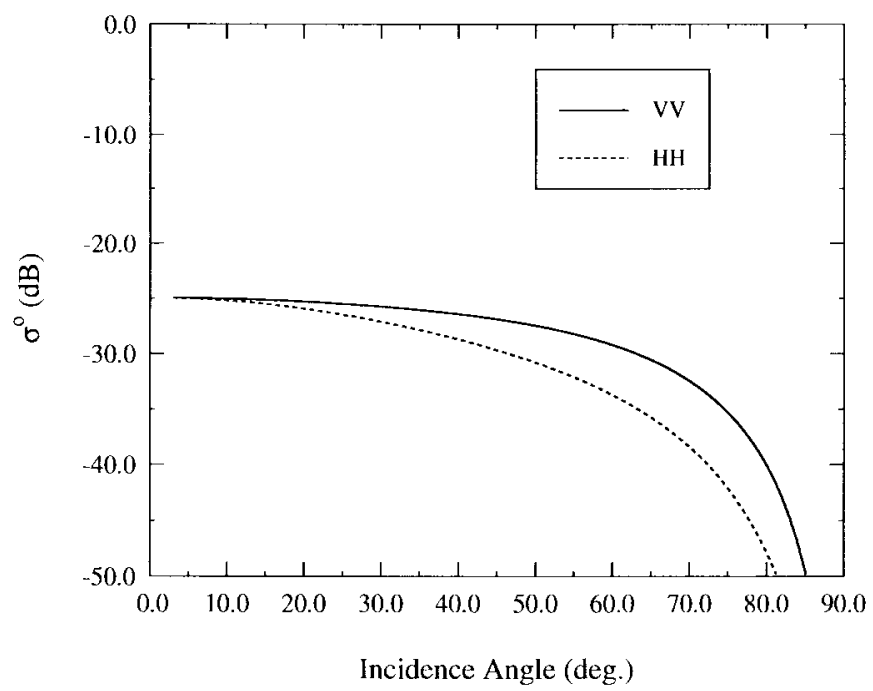

Fig. 4. Predictions of surface scattering for 8-12 gravel, generated using the SPM.

The importance of the influence of the surface backscatter on the measurements may be gauged to some degree by the variability of the signal with different target parameters. If, for example, at a given incidence angle, backscatter changes very little with changing depth, or with changes in the underlying "halfspace" material, it may be suspected that the dominant source of the backscatter is the surface term. As will be shown in the following sections, this was not found to be the case in our measurements. However, even for the case in which the signal is seen to be quite responsive to changes in the target configuration, it is possible that the small surface term may represent a substantial contribution for the cases involving the very lowest backscatter levels, and so introduce errors into an analysis based on volume scattering exclusively. For this reason, an attempt was made to calculate the expected backscatter based on measured parameters of the surface.

The surface parameters consist of the effective dielectric constant, given in Fig. 3, and the roughness parameters, rms height, and correlation length, which can be obtained through the measurement of the surface height profile. Accordingly, the surface height profiles were measured with a laser profiler. The rms height $s$ was found to be $0.66 \mathrm{~mm}$, corresponding to a value $k s=0.022$. The autocorrelation function $l$ was seen to be essentially exponential, with a correlation length of about $2 \mathrm{~mm}$, for which $k l=1.18$.

A suitable theoretical solution for these parameters is given by the small perturbation method (SPM) (see, for example, [19]). The estimated backscatter as a function of angle is presented in Fig. 4. As anticipated, the backscattering level predicted is quite low for this smooth surface. The relative contribution of this surface scattering component may be evaluated with respect to the measurements that are presented next.

\section{INTERPRETATION OF RESULTS}

In the following sections, we present the results of our controlled experiments and analyze them in the context of the hybrid modeling approach outlined in Section II. It is our intent to investigate whether the Rayleigh approach described in Section III constitutes an appropriate polarimetric model for the test material being considered.

Before proceeding further, however, it will first be instructive to state explicitly what constitutes a comprehensive comparison between a polarimetric model and measurements. For an azimuthally symmetric medium, there are normally considered to be five independent quantities contained in the measured Mueller matrix in the backscatter direction. These are the copol responses $\left|S_{v v}\right|^{2}$ and $\left|S_{h h}\right|^{2}$, the cross-pol response $\left|S_{v h}\right|^{2}$ (=|Shv $\left.\right|^{2}$ for backscatter), and two parameters that together specify the statistics of the copolarized phase difference $\phi_{h h}-\phi_{v v}$. The pdf governing this random variable has been shown to be [21]

$$
\begin{aligned}
f_{\Phi}(\phi)= & \frac{1-\alpha^{2}}{2 \pi\left[1-\alpha^{2} \cos ^{2}(\phi-\zeta)\right]} \\
& \times\left\{1+\frac{\alpha \cos (\phi-\zeta)}{\sqrt{1-\alpha^{2} \cos ^{2}(\phi-\zeta)}}\right. \\
& \left.\times\left[\frac{\pi}{2}+\tan ^{-1} \frac{\alpha \cos (\phi-\zeta)}{\sqrt{1-\alpha^{2} \cos ^{2}(\phi-\zeta)}}\right]\right\}
\end{aligned}
$$

which is specified by the two parameters $\alpha$-the degree of correlation - and $\zeta$ - the copolarized phase difference. The quantities are defined in terms of the elements of the Mueller matrix as

$$
\alpha=\sqrt{\frac{\lambda_{13}^{2}+\lambda_{14}^{2}}{\lambda_{11} \lambda_{33}}}, \quad \zeta=\tan ^{-1} \frac{\lambda_{14}}{\lambda_{13}}
$$

where

$$
\begin{aligned}
\lambda_{11} & =\frac{\mathcal{M}_{11}}{2}, & \lambda_{33} & =\frac{\mathcal{M}_{22}}{2} \\
\lambda_{13} & =\frac{\mathcal{M}_{33}+\mathcal{M}_{44}}{4}, & \lambda_{14} & =\frac{\mathcal{M}_{34}-\mathcal{M}_{43}}{4}
\end{aligned}
$$

where $\mathcal{M}_{p q}$ are elements of the Mueller matrix. Therefore, a comprehensive comparison will examine the agreement between the model and the data with respect to these five elements.

Extensive data were collected for the 8-12 material introduced above, using the experimental procedure described in Section IV. The 8-12 gravel was examined for ten total layer depths - four over a conductor and six over an absorbing layer (except at $30^{\circ}$, for which it was only two depths over an absorbing layer and five over a conductor) - for incidence angles ranging from 20 to $60^{\circ}$. There was some indication in the results, however, not predicted by the surface scattering analysis done in the previous section, that at $20^{\circ}$ there was some surface contribution to the backscatter that was not negligible. Therefore, the $8-12$ gravel is analyzed using only the data corresponding to 30,40 , and $60^{\circ}$.

\section{A. Comparison with Rayleigh Model}

The Rayleigh model derived in Section III utilized a scalar extinction and phase matrix components $\mathcal{P}_{b s}$ and $\mathcal{P}_{b i}$ given by (25) and (26). As mentioned, these phase matrix components 


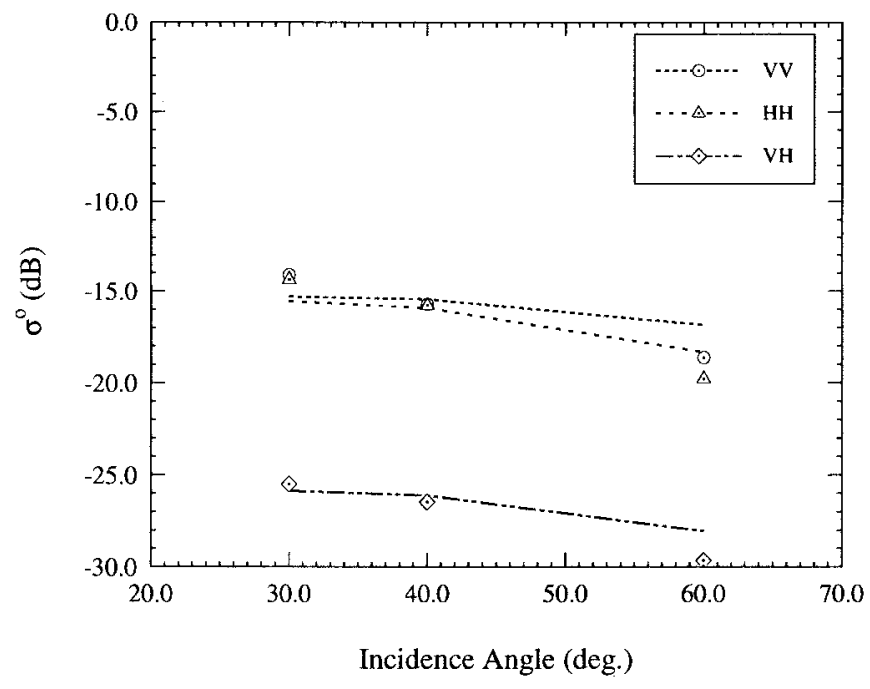

(a)

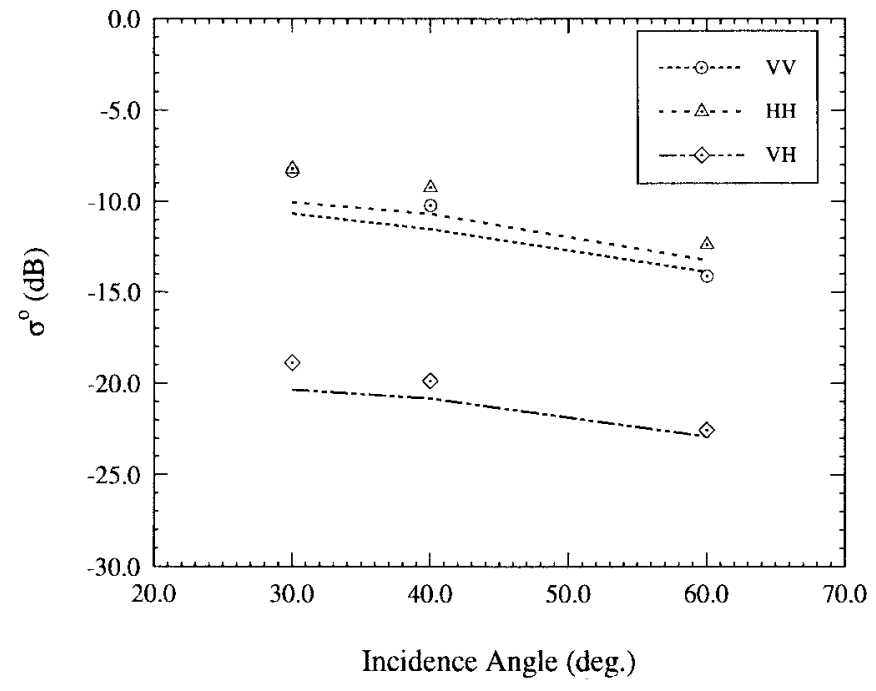

(b)

Fig. 5. Application of Rayleigh model to measured data. Comparison for copolarized and cross-polarized response, over both (a) absorber and (b) conductor for 8-12 gravel.

were entirely specified by just two parameters, which are themselves functions of the elements of the polarizability tensor of the arbitrary Rayleigh particles. This theoretical framework was used along with the results from the measurements in the inversion algorithm described in Section V. Some selected results of this analysis are shown in Figs. 5 and 6. These results are representative of the degree of success that was achieved generally in comparing the Rayleigh model to the measured data. Fig. 5 shows the angular variation of the copol and crosspol responses, examined over both a conductor and an absorber at the depths specified in the figures. In general, the angular variation built into the Rayleigh model does not agree with the observed behavior very well.

Fig. 6 presents the results with respect to the phase statistics. Only the degree of correlation $(\alpha)$ is shown at specific layer depths over both absorber and conductor. It is clear that the Rayleigh model is inadequate for explaining the observed behavior for these materials. The lack of success of this model

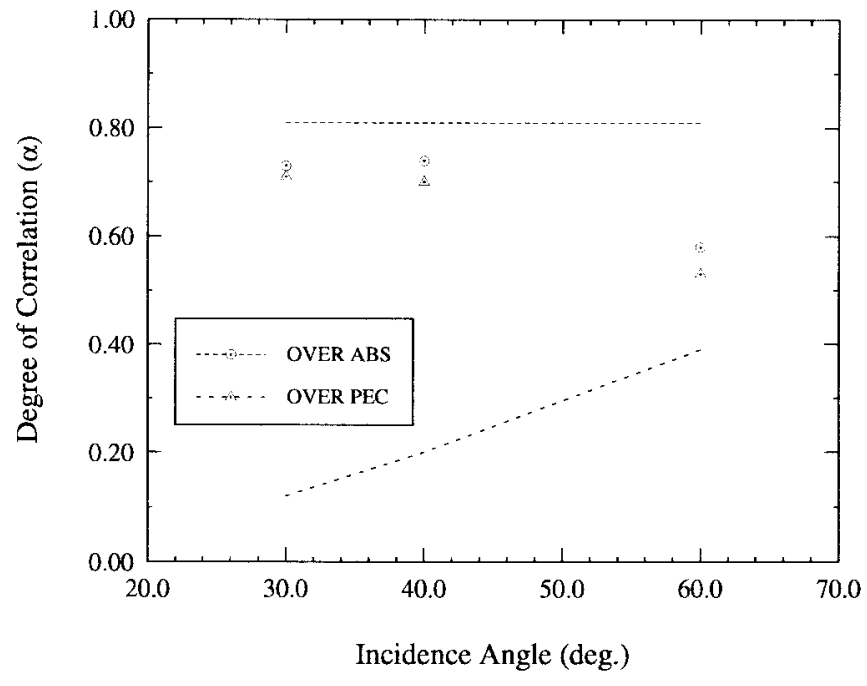

Fig. 6. Application of Rayleigh model to measured data. Comparison for degree of correlation $(\alpha)$. Layers over absorber and conductor are 14.3 and $16.5 \mathrm{~cm}$, respectively.

is not too surprising. As mentioned, the individual particles in the medium are for the most part pushing the limit of Rayleigh particles for the wavelength being used. When consideration is made of some "effective" aggregate particle, which might comprise many individual particles, the hypothesis becomes even more doubtful. Still it is possible that such a model might be valid for smaller particles and where the volume fraction is not quite so high.

The angular variation in the Rayleigh model is controlled by two factors: 1) the Fresnel transmissivity of the surface and 2) the explicit dependence shown in (26). It is possible that a general isotropic model could work if the explicit constraints of the Rayleigh model are relaxed.

\section{B. General Isotropic Model}

For the general isotropic model, the quantities of interest take the following forms.

- Diagonal elements of the extinction matrix are required to be identical. This is easily understood from the very definition of isotropic media. The propagation characteristics of the medium are insensitive to the polarization of the intensity. Thus, the extinction for vertical polarization must be the same as for horizontal. For the nondiagonal elements of the extinction matrix, which for general nonspherical particles is given in [10], we assume the cross coupling between $\mathbf{v}$ and $\mathbf{h}$ polarizations is zero for forward scattering, making these off-diagonal elements zero. Thus, for the general isotropic model we are considering, the extinction $\kappa_{e}$ becomes a scalar $\kappa_{e}$, as in the Rayleigh model.

- The form of $\mathcal{P}_{b s}$ also resembles that of the Rayleigh model. The isotropic constraint requires that $\left\langle S_{v v} S_{v v}^{*}\right\rangle=\left\langle S_{h h} S_{h h}^{*}\right\rangle$. Reciprocity requires that $S_{v h}=S_{h v}$ [in the backscattering alignment (BSA); in the forward scattering alignment, $\left.S_{v h}=-S_{h v}\right]$. From arguments of azimuthal symmetry and experimental evidence, the copol and the cross-pol terms have been 
found to be statistically uncorrelated; thus, all terms of the form $\left\langle S_{p p} S_{p q}^{*}\right\rangle$ are zero. The resulting form of the matrix, which is independent of incidence angle, is

$$
\mathcal{P}_{b s}=\left[\begin{array}{cccc}
P_{2} & P_{3} & 0 & 0 \\
P_{3} & P_{2} & 0 & 0 \\
0 & 0 & P_{4}-P_{3} & P_{\tilde{5}} \\
0 & 0 & -P_{5} & P_{4}+P_{3}
\end{array}\right] .
$$

- For the $\mathcal{P}_{b i}$ matrix, there is less that can be specified in advance. Reciprocity does not require the $S_{v h}$ and $S_{h v}$ terms to be identical, and the like- and cross-pol terms are not necessarily uncorrelated. For the latter point, we will borrow from the findings of the Rayleigh analysis, which showed these covariance terms to be zero, but relieve generally the other constraints placed on the elements of this matrix. In particular, we will allow the matrix as a whole to be a function of the incidence angle, as specified by $\mu_{o}\left(\mu_{o}=\cos \theta_{0}\right)$. The structure is

$$
\mathcal{P}_{b i}=\left[\begin{array}{cccc}
P_{6} & P_{7} & 0 & 0 \\
P_{8} & P_{9} & 0 & 0 \\
0 & 0 & P_{10} & P_{11} \\
0 & 0 & P_{12} & P_{13}
\end{array}\right] .
$$

Mathematically, the resulting model is identical to that given in (4), using $\mathcal{P}_{b s}$ and $\mathcal{P}_{b i}$ as given above. There are 13 parameters to be specified for a complete polarimetric description for a single angle. Of these, the extinction $\kappa_{e}$ and the matrix $\mathcal{P}_{b s}$ (comparing five parameters) will be common to all angles. A separate bistatic matrix $\mathcal{P}_{b i}$ must be determined for each angle.

Thirteen parameters is a considerable space to explore for an algorithm for nonlinear optimization. Given the task of optimizing all 13 parameters at once, the algorithm will generally not produce a very satisfactory result, and it will tend to arrive at different solutions depending on the initial guess. Fortunately, it is not necessary to optimize all parameters simultaneously. The following option exists for a much more limited optimization process.

First, it is recognized that, if a "quadrant" is defined by a $2 \times 2$ submatrix, proceeding clockwise from the top left of the $4 \times 4$ matrices in this formulation, the first and third quadrants are decoupled from one another since the second and fourth quadrants are identically zero. The zero status of these latter two quadrants holds for all of the matrices involved in the first-order solution, including the transmissivity and reflectivity matrices. Thus, it is possible to optimize initially only the seven parameters affecting the copol and cross-pol responses. After these have been found, the remaining six may be found, using the value for $\kappa_{e}$ obtained in the initial process. Additional iterations may be performed to improve the overall result. In general, it is found that, due to a model that is only an approximation and data that contains errors, no absolute convergence is observed from an iterative process, and degradation of the perceived "goodness" of the solution occurs with many iterations.

A complete comparison of the isotropic modeling approach with the measured data is shown in Figs. 7-9 for the 8-12 gravel, for incidence angles 30,40 , and $60^{\circ}$. All of the available data were used in the inversion process suggested by (27) to find the optimal parameters for the first-order polarimetric model given by (4). Each of the figures constitutes a comprehensive comparison of the five aforementioned independent elements of the measured Mueller matrix with the model predictions for the angle specified.

The agreement with the $8-12$ gravel is seen to be generally very good. The copolarized and cross-polarized response for layers over a conducting surface [(a) in Figs. 7-9] is very well modeled. There is some small disagreement for the case of the copol response over an absorber layer, at all three angles. The response is somewhat underpredicted (by $1-2 \mathrm{~dB}$ ) at $30^{\circ}$ and over predicted by about this amount at $60^{\circ}$. This is an interesting and important case to examine since the bistatic component of the phase function $\mathcal{P}_{b i}$, which can be individually tailored for each incidence angle in the inversion process, is not involved. Since the backscatter component $\mathcal{P}_{b s}$ is a constant quantity, the angular variation is governed solely by the Fresnel transmissivity of the surface. Angular behavior that is in agreement with this Fresnel variation, for a "halfspace," which our layer-over-absorber approximates, is a necessary requirement for scattering that is truly first order and isotropic.

The behavior of the phase statistics of the measurements appears to be a very simple function of all of the parameters varied (angle, depth, underlying "halfspace"), and it is modeled fairly well by this first-order hybrid model. The parameter $\zeta$, the copolarized phase difference is, in particular, explained by the model quite well at all three angles.

\section{COMPARISON OF RESUltS WiTH THEORY}

In Section VI, we stated that the characteristics of the 8-12 gravel made it especially suitable for comparison with theory. One impediment, however, to such a comparison is the task of determining the precise dielectric constant of the particles themselves $\left(\tilde{\epsilon}_{p}\right)$ at the frequency of interest, in this case, our center frequency of $16 \mathrm{GHz}$.

One approach by which this might be accomplished is to measure by some means the effective dielectric constant $\tilde{\epsilon}_{\mathrm{eff}}$ of the medium and then invert a dielectric mixing formula like the Polder-Van Santen mixing model [19] to get the dielectric of the inclusions (particles). The fact that the particles have a high degree of sphericity removes the ambiguity associated with assigning the three shape factors for that model. If, however, $\tilde{\epsilon}_{\text {eff }}$ is measured at the test frequency $(16 \mathrm{GHz}$ was the center frequency), the imaginary part $\epsilon_{\mathrm{eff}}^{\prime \prime}$ may comprise the effects of the scattering losses as well as the dielectric conducting losses, depending on the measurement technique. If the scattering losses are included in the measurement of $\tilde{\epsilon}_{\mathrm{eff}}$, inversion of a dielectric mixing model will not lead to the proper result for the particle dielectric $\tilde{\epsilon}_{p}$. A complex reflection coefficient waveguide technique might be employed at this frequency $(16 \mathrm{GHz})$ in which (it may be argued) scattering losses are prevented due to the boundaries (i.e., modal requirements) of the guide; this point, however, is at this time still something of a research question, and in any event, the technique is prone to error when the loss tangent of the test material is very small.

A more appropriate procedure is to measure $\epsilon_{\mathrm{eff}}^{\prime \prime}$ at low frequency, in which scattering is negligible, independent of 


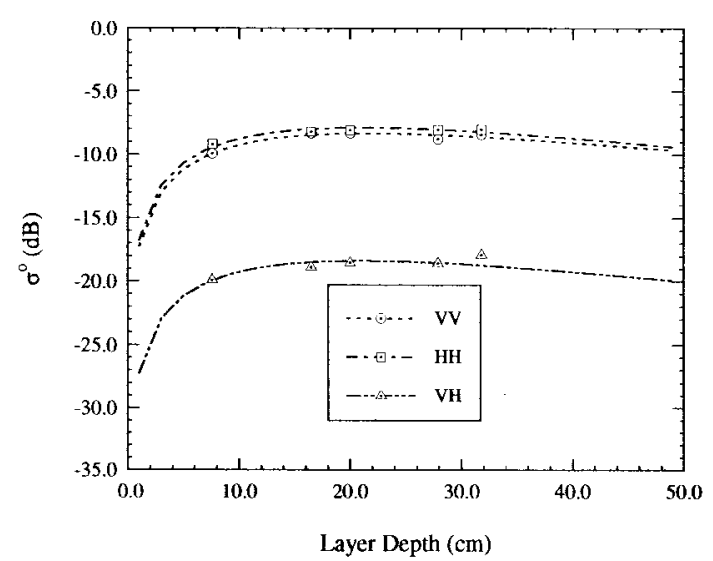

(a)

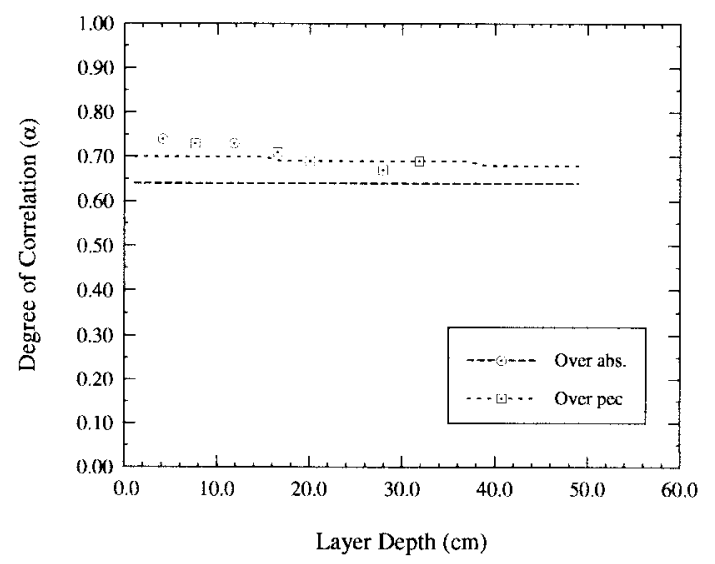

(c)

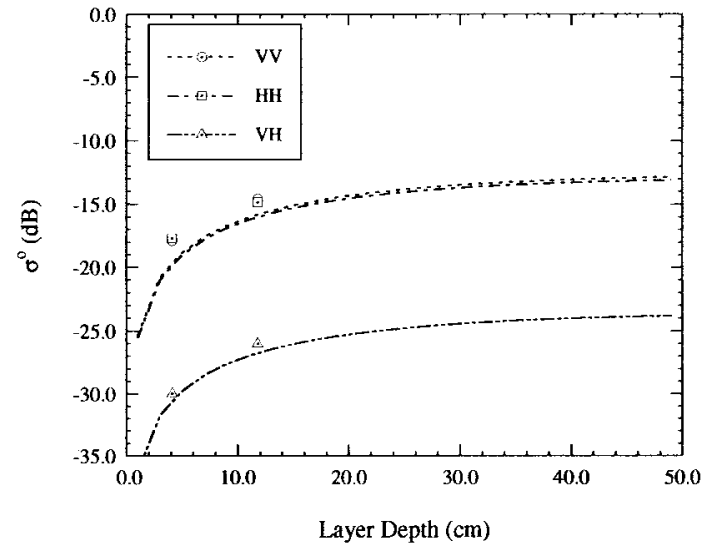

(b)

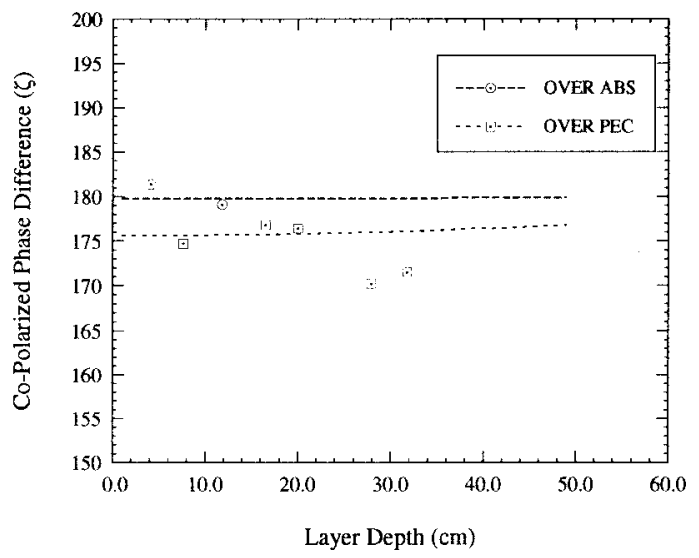

(d)

Fig. 7. Results of application of hybrid RT model to measured data: 8-12 gravel, $30^{\circ}$ incidence [(a) conductor and (b) absorber underlying layer].

the measurement technique used. The variation of the dielectric constant of dry rocks with frequency in the microwave region has been studied [22]. The real part $\epsilon_{p}^{\prime}$ is essentially constant from 1 to $16 \mathrm{GHz}$. The imaginary part $\epsilon_{p}^{\prime \prime}$ has been found to decrease with frequency, in a manner dependent on the particular rock class. The material in the 8-12 gravel appears to be either a sedimentary, plutonic, or volcanic silicate. The frequency dependance of $\epsilon_{p}^{\prime \prime}$ for each one of these classes is given in [22].

In this case, $\tilde{\epsilon}_{\text {eff }}$ for the $8-12$ gravel was measured at about $1.5 \mathrm{GHz}$ using the Snow Probe (described in [18]) and found to be $\tilde{\epsilon}_{\mathrm{eff}}=2.17-j 0.007$. The Polder-Van Santen mixing formula for spherical particles (shape factors: $A_{1}=A_{2}=$ $\left.A_{3}=1 / 3\right)$ is

$$
\epsilon_{m}=\epsilon_{h}+3 \nu_{i} \epsilon_{m} \frac{\epsilon_{i}-\epsilon_{h}}{\epsilon_{i}+2 \epsilon_{m}}
$$

where $\epsilon_{m}, \epsilon_{h}$, and $\epsilon_{i}$ are the effective dielectric constants of the medium, the host (air), and the inclusions (or particles), respectively, and $\nu_{i}$ is the volume fraction. This equation may be easily solved for $\epsilon_{i}\left(=\tilde{\epsilon}_{p}\right)$, yielding $3.11-j 1.38 \times 10^{-2}$.

This value of $\epsilon_{p}^{\prime \prime}$ is already considerably lower than the average for all of the silicate classes mentioned above (see Fig. 13 in [22]), although still within the appreciable scatter of the data. Therefore, instead of applying the frequency dependance associated with the much higher values of the quantity $\epsilon_{p}^{\prime \prime}$ measured for the various silicate classes in [22], we will simply use the low-frequency value of $\tilde{\epsilon}_{p}$ as our estimate at the frequency of interest, $16 \mathrm{GHz}$. Since, in general, the study found that $\epsilon_{p}^{\prime \prime}$ decreases with frequency, the use of this low-frequency result will lead to a maximum attenuation calculation and a minimum scattering calculation.

This result completes the information required to examine the correspondence between the measurements of this material and the predictions of certain discrete particle-based theories. A pertinent quantity for comparison is the effective propagation constant in the medium

$$
K=K^{\prime}-j K^{\prime \prime}
$$

from which may be obtained the real and imaginary parts of the effective index of refraction

$$
n^{\prime}=\frac{K^{\prime}}{k_{\mathrm{o}}}, \quad n^{\prime \prime}=\frac{K^{\prime \prime}}{k_{\mathrm{o}}}
$$

with the extinction specified as $\kappa_{e}=2 K^{\prime \prime}$.

The theories we compare with are the effective field approximation (EFA), the quasicrystalline approximation (QCA), and the quasicrystalline approximation with coherent potential (QCA-CP). We present here a very brief description of each technique.

The EFA (also known as Foldy's approximation) can be considered a special case of QCA [14], in that the particles are 


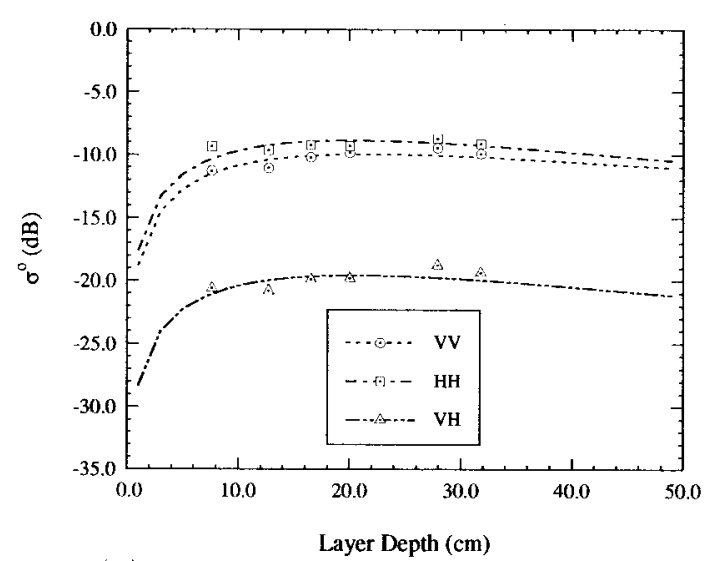

(a)

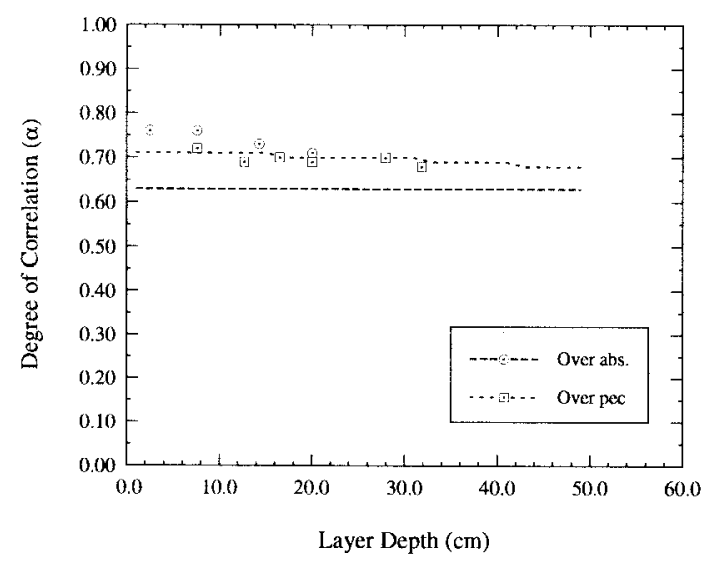

(c)

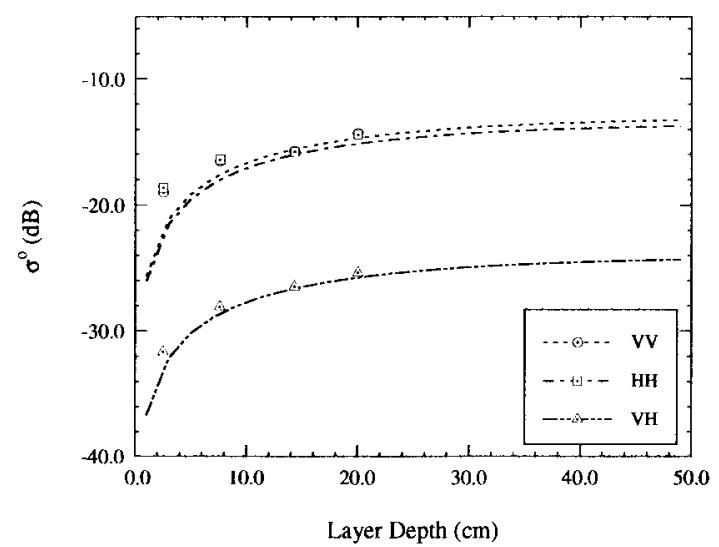

(b)

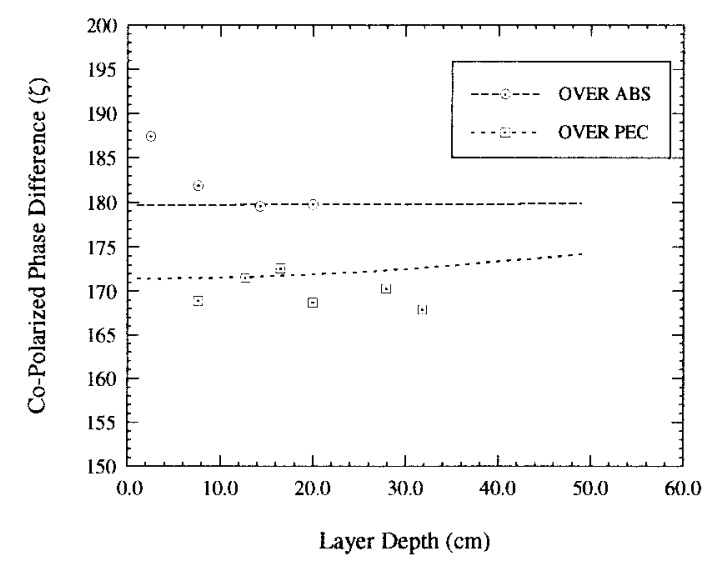

(d)

Fig. 8. Results of application of hybrid RT model to measured data: 8-12 gravel, 40 incidence [(a) conductor and (b) absorber underlying layer].

considered as acting completely independent of one another with zero correlation. It leads to an attenuation rate that is very similar to that of independent scattering [14, p. 427]. The solution for the effective propagation constant is formally given by [23]

$$
K=\left[k^{2}-4 \pi n_{\circ}<S_{p q}\left(\theta_{s}, \phi_{s} ; \theta_{i}, \phi_{i}\right)>\right]^{1 / 2}
$$

where $S_{p q}$ is the complex far-field scattering amplitude, $k$ is the wavenumber of the surrounding medium, and $n_{0}$ is the number density of the scatterers.

A detailed description of QCA theory is outside the scope of this presentation. A detailed derivation may be found in [14]. In general, QCA takes into account interactions between particles, using exact wave transformations, a "T-matrix" approach, for computing this interparticle interaction rather than farfield phase functions. The particle correlation is specified by the pair-distribution function, which describes the conditional probability of a particle location relative to another particle's position. The Percus-Yevick approximation is most often used to derive the pair-distribution function for a medium. This approximation assumes noninterpenetrability of particles and zero forces between the particles. This assumption, which has, in some specific experiments [23], been found to agree with experimental observations, removes the requirement of characterizing by more direct means the exact configuration of particles in a dense medium. In more recent studies [24], this assumption has been shown to breakdown outside of its intended domain of validity, which comprises mainly liquids or gases.

QCA-CP constitutes an improvement on QCA in that it provides for energy conservation in the formulation. Essentially, it amounts to employing the effective wavenumber $K$ in the Green's functions of the QCA formulation instead of the background wavenumber $k$. The formulation is valid for Rayleigh particles only.

The results produced by each one of these methods are summarized in Table II. Shown are estimates for $n^{\prime}$, the real part of the effective index of refraction and $\kappa_{e}$. Shown for comparison are the snow probe measured $n^{\prime}$ and $\kappa_{e}$ obtained from the hybrid model analysis of the 8-12 gravel. The correlated particle treatments represented by QCA and QCA-CP produce estimates of $n^{\prime}$, which are in close agreement with the measured value. EFA gives a considerably lower estimate. While the QCA result for $\kappa_{e}$ is very low, the QCA-CP and EFA estimates bracket, below and above, respectively, the experimentally determined value for $\kappa_{e}$. This bracketing scenario resembles previous findings [23] for glass spheres in styrofoam at much lower volume fractions $(<10 \%)$.

Fig. 10 shows a comparison between measurements and scattering computations from a numerical CRT model that employs the EFA theory, using Mie calculations for the phase function and extinction and the discrete ordinate solution 


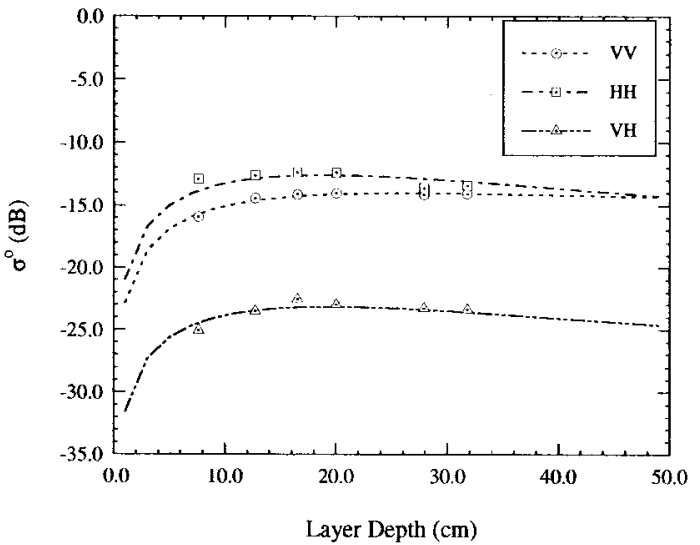

(a)

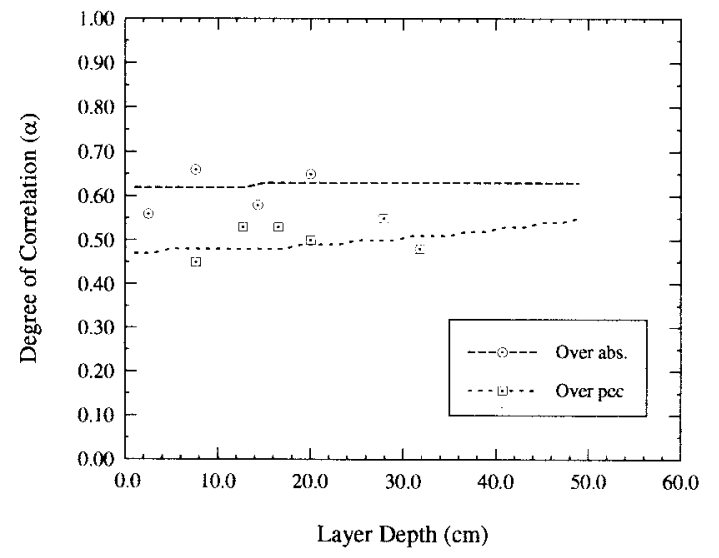

(c)

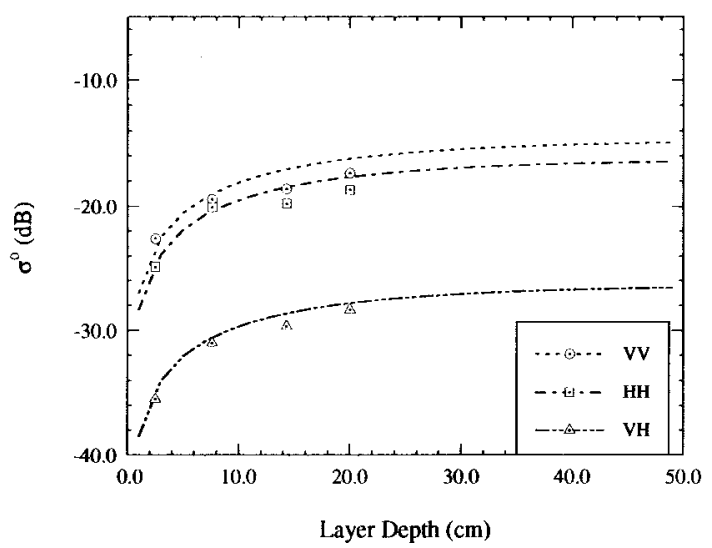

(b)

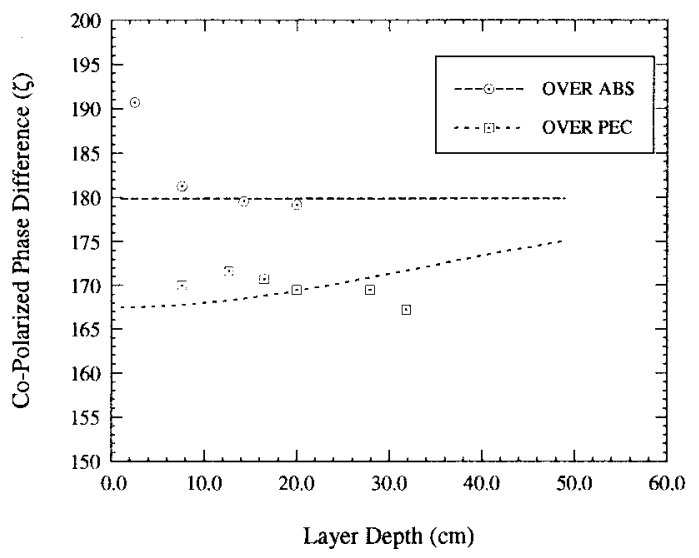

(d)

Fig. 9. Results of application of hybrid RT model to measured data: 8-12 gravel, $60^{\circ}$ incidence [(a) conductor and (b) absorber underlying layer].

TABLE II

Propagation Parameters for 8-12 Gravel

\begin{tabular}{l|c|c}
\hline \hline Source & $n^{\prime}$ & $\kappa_{e}$ \\
\hline Snow Probe & 1.47 & --- \\
\hline Iybrid Model & --- & 2.35 \\
\hline EHA & 1.35 & $3.09 / 3.02^{*}$ \\
\hline QCA & 1.43 & 0.29 \\
\hline QCA-CP $^{\circ}$ & 1.47 & 1.69
\end{tabular}

${ }^{*}$ Background $=$ air / Background $=\epsilon_{e f f}$

${ }^{\circ}$ Intended for Rayleigh region.

method [25]. The inputs to the model consisted of the physical characteristics of the 8-12 gravel, as they have been described in the preceding sections, including particle size distribution, volume fraction, effective dielectric constant of the medium, and dielectric constant of the individual particles. Though the trends with respect to polarization and depth are similar, the CRT model overestimates the scattering level by typically 3-4 dB. Recalling that the value of the imaginary part of the particle dielectric used is a maximum value, this discrepancy would be expected to increase with a more accurate estimation of $\epsilon_{p}$, as the single scattering albedo of the particle would increase.

A comparison between measurements and predictions from a dense medium radiative transfer (DMRT) model, described in Section II, as shown in Fig. 11. The theoretical predictions are seen to be extremely low-on the order of $15-20 \mathrm{~dB}$-relative to the measured results. This very serious disagreement can be explained in terms of the behavior of the scattering albedo $\omega_{0}$, which scales the phase matrix (as described in Section II), and hence the scattering production of a collection of scatters. The value of $\omega_{0}$ computed (from [8]) for the DMRT model is similar to the CRT computation for very low volume fractions (e.g., $<5 \%$ ) but diminishes rapidly with increasing volume fraction. For this scenario, with a volume fraction of 0.63 , the scattering albedo computed from DMRT is $5.1 \times 10^{-3}$, about 250 times smaller than that computed by CRT. Since the values of extinction given in Table II for EFA and QCA-CP are fairly similar, this means that the difference in the respective albedos pertains to the scattering (recall, $\omega_{0}=\kappa_{s} / \kappa_{e}$ ). In short, at these volume fractions, the DMRT predicts negligible scattering production from the medium. The value for the extinction computed from QCA-CP given in Table II is almost entirely associated with the absorptive losses in the particles. That the QCA result is smaller than this value is apparently symptomatic of that technique's failure to obey energy conservation. We note also that the issue of an extremely diminished scattering albedo makes noncritical the question of a more precise determination of the particle dielectric.

The diminishment of the scattering albedo in the DMRT formulation stems from the nature of the pair distribution 


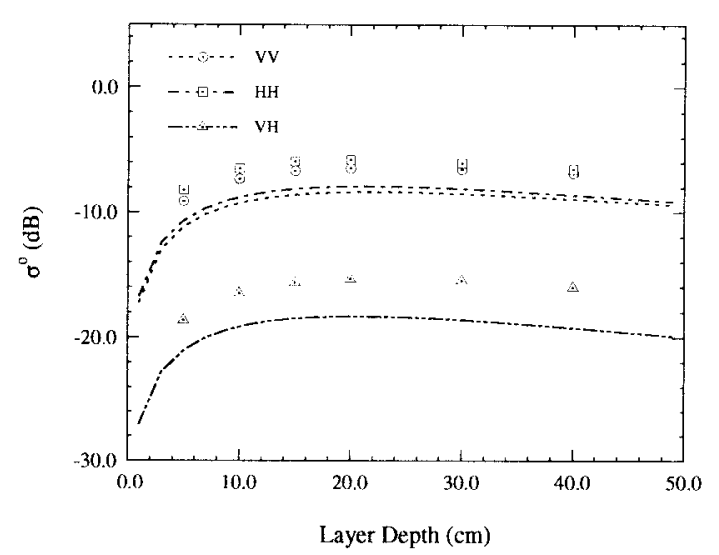

(a)

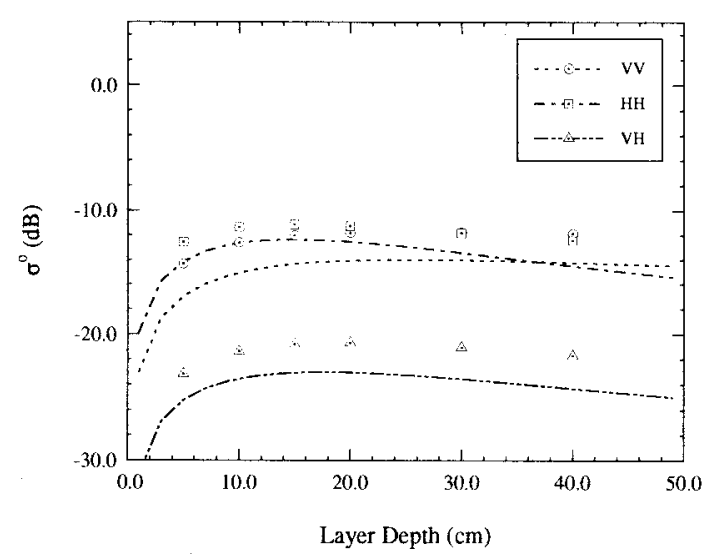

(c)

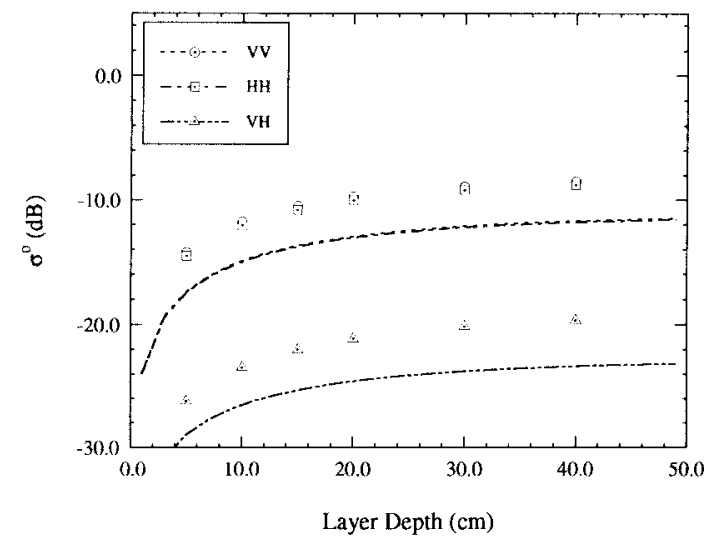

(b)

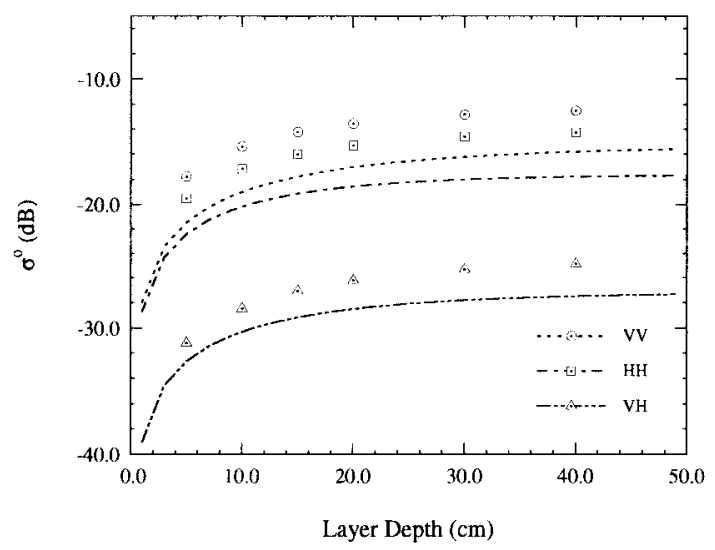

(d)

Fig. 10. Comparison of measurements of 8-12 gravel with CRT simulations. Discrete marks are CRT simulations; continuous lines are from hybrid model developed from analysis of measured data, which very closely approximates the actual data [(a) $30^{\circ}$ incidence over conductor, (b) $30^{\circ}$ incidence over absorber, (c) $60^{\circ}$ incidence over conductor, and (d) $60^{\circ}$ incidence over absorber].

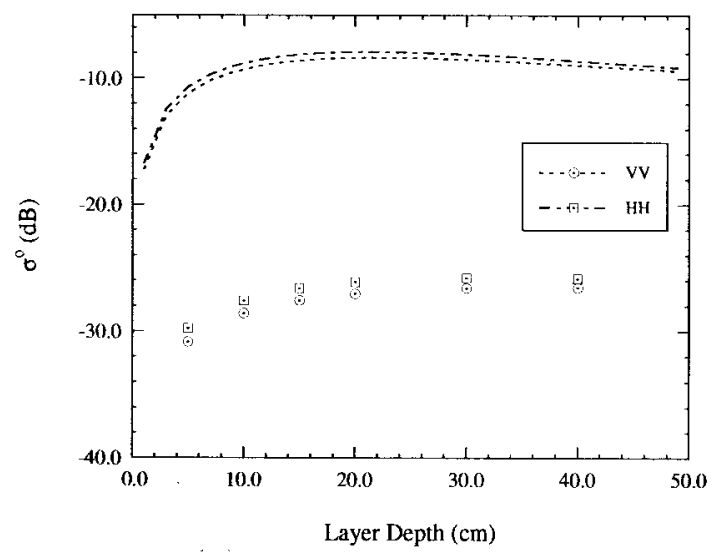

(a)

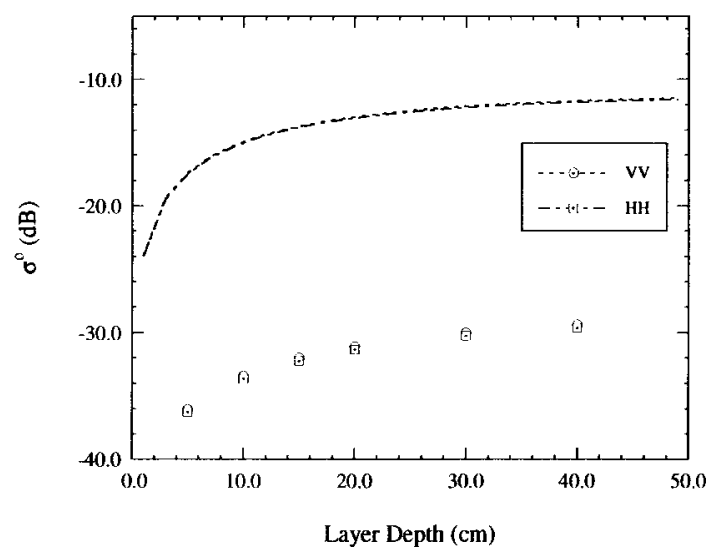

(b)

Fig. 11. Comparison of measurements of 8-12 gravel with DMRT simulations at $30^{\circ}$ incidence. Discrete marks are DMRT simulations; continuous lines are from hybrid model developed from analysis of measured data, which very closely approximates the actual data [(a) over conductor and (b) over absorber].

function as computed under the Percus-Yevick approximation. For a volume fraction as high as that of the material under test, 0.63 , the Percus-Yevick pair distribution function predicts a great deal of order in the system of particles. This is depicted in Fig. 12, where the function is shown for the current volume fraction (0.63) as well as for a much smaller volume fraction $v=0.25$. At the 0.63 volume fraction, near the maximum packing density for spheres, it is seen that a fairly orderly, crystal-like system is predicted with alternating regions of very high and low probability of neighbor particles. The formulation itself appears to begin to breakdown at this point, as evidenced by certain irregular-looking features, in contrast with the appearance of the function at $v=0.5$, also shown in the figure. 


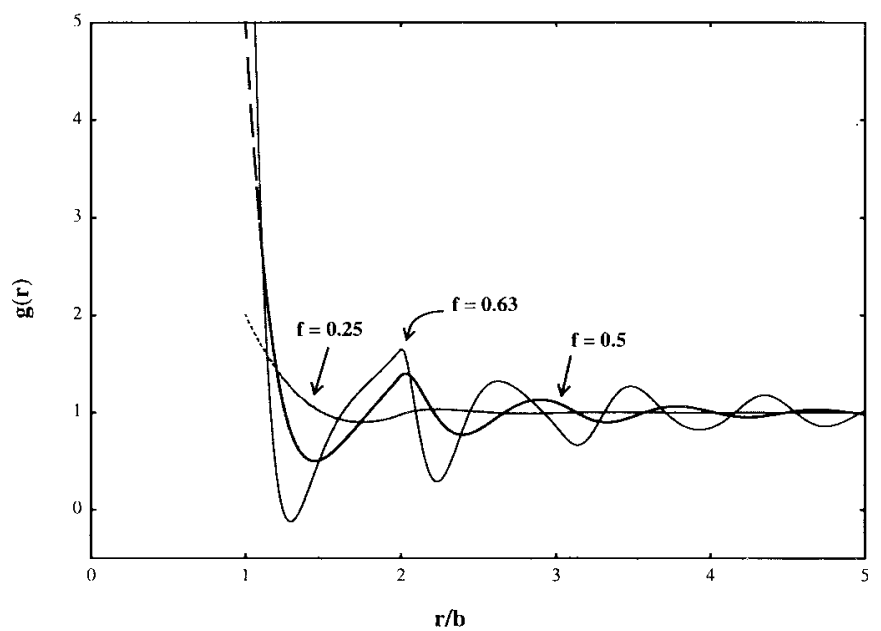

Fig. 12. Percus-Yevick pair distribution function for volume fractions $v=0.63, v=0.5$, and $v=0.25$.

For a final comparison, we note that in the hybrid model, finding $\mathcal{P}_{b i}$ independently for various incidence angles amounts to mapping out some portion of the scattering phase function of the particle (refer, for example, to Fig. 1). Fig. 13 shows these experimentally derived phase functions. Shown for comparison are Mie calculations made for the cases of 1) a particle having the same characteristics as the 8-12 gravel particles and 2) a particle having the same dielectric but three times as large. All results are normalized to the backscatter level. The obvious implication, consistent with the assumptions of the hybrid model concept, is that multiple particles combine to form an effective scattering element that is amenable to a first-order RT approach.

\section{Summary AND CONClusions}

This paper has described a hybrid experimental/theoretical technique by which dense medium scattering may be modeled. Specifically, we have demonstrated that the complete polarimetric backscatter response from a very dense medium consisting of discrete particles can be adequately explained in terms of a vector first-order RT model.

Very simple forms of the phase matrix components involved in the first-order formulation have been derived using Rayleigh theory. While these specific formulations were not observed to correctly model the experimentally observed results in the present case, it is possible they may still be valid if applied to a somewhat less dense medium with smaller particles.

The dense medium backscatter measurements were compared against predictions from conventional and dense medium RT. While the CRT results were reasonably close, the scattering predicted by DMRT for this high volume fraction material $\left(v_{i}=0.63\right)$ was $15-20 \mathrm{~dB}$ below the measured levels.

Finally, application of the hybrid technique to direct measurements though a numerical optimization algorithm also yields a partial mapping of the scattering phase function of the effective volume scattering element in the medium. An analysis has been presented that suggests that this element is considerably larger than the physical particles of which the medium is comprised.

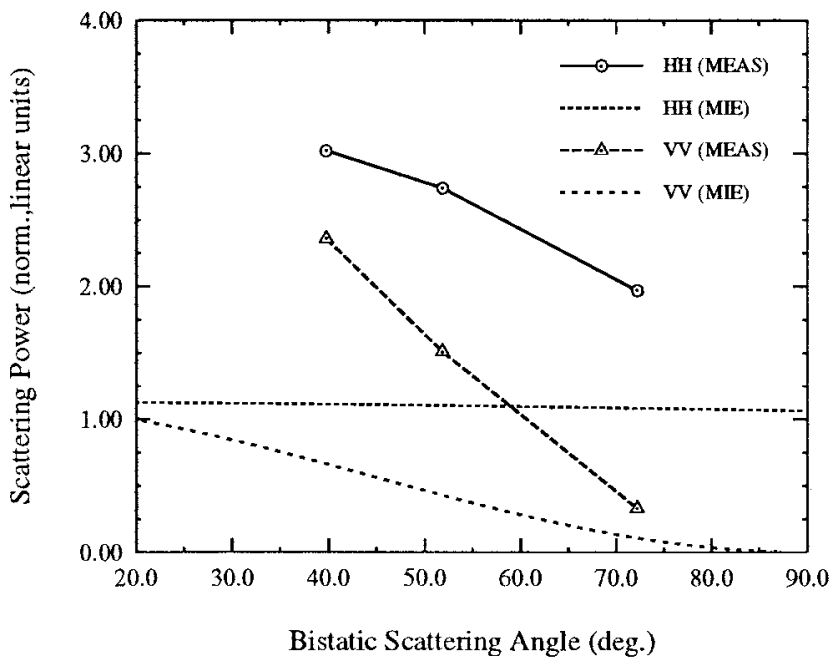

(a)

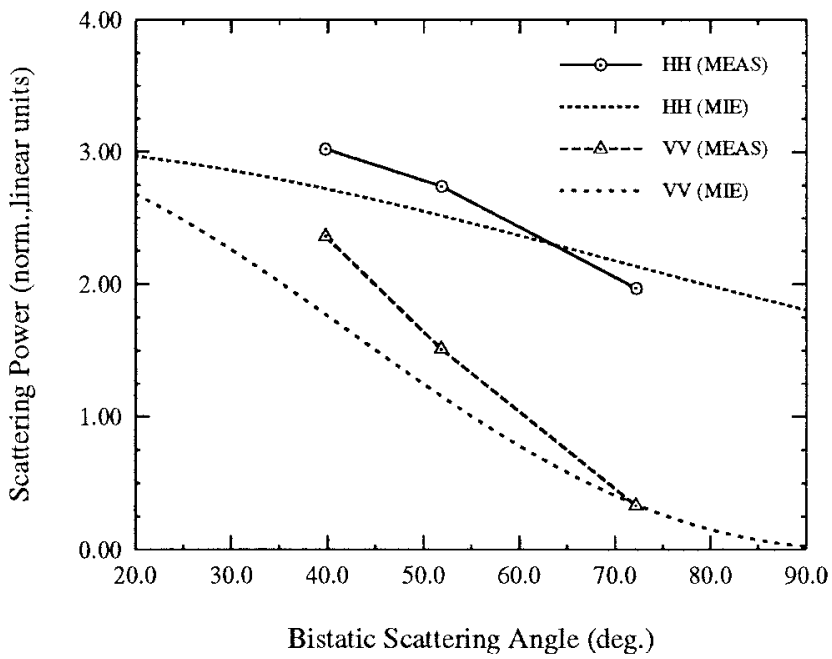

(b)

Fig. 13. Portion of the scattering phase function obtained via isotropic RT analysis of 8-12 gravel compared with Mie phase function for a particle (a) identical to an 8-12 gravel particle and (b) three times larger than an 8-12 gravel particle.

\section{REFERENCES}

[1] A. Nashashibi and K. Sarabandi, "Propagation in dense random media: A novel measurement technique and experimental results," IEEE Trans. Antennas Propagat., to be published.

[2] J. R. Kendra, K. Sarabandi, and F. T. Ulaby, "Radar measurements of snow: Experiment and analysis," IEEE Trans. Geosci. Remote Sensing, vol. 36, pp. 864-879, May 1998.

[3] F. Vallese and J. A. Kong, "Correlation function studies for snow and ice," J. Appl. Phys., vol. 52, pp. 4921-4925, Aug. 1981.

[4] G. Koh and R. E. Davis, "Effect of snow stereology on millimeter wave extinction," in Proc. IGARSS'94, vol. 4, p. 1929.

[5] P. R. Siqueira, K. Sarabandi, and F. T. Ulaby, "Numerical simulation of scatterer positions in a very dense medium with an application to the two-dimensional born approximation," Radio Sci., vol. 30, no. 5, pp. 1325-1339, 1995.

[6] L. Tsang and A. Ishimaru, "Radiative wave equations for vector electromagnetic propagation in dense nontenuous media," J. Electromagn. Waves Applicat., vol. 1, no. 1, pp. 59-72, 1987.

[7] F. T. Ulaby and C. Elachi, Eds., Radar Polarimetry for Geoscience Applications. Dedham, MA: Artech House, 1990.

[8] B. Wen, L. Tsang, D. P. Winebrenner, and A. Ishimaru, "Dense medium radiative transfer theory: Comparison with experiment and application 
to microwave remote sensing and polarimetry," IEEE Trans. Geosci. Remote Sensing, vol. 28, pp. 46-59, Jan. 1990.

[9] L. Tsang and J. A. Kong, "Scattering of electromagnetic waves from a dense medium consisting of correlated Mie scatters with size distributions and applications to dry snow," J. Electromagn. Waves Applicat., vol. 6, no. 3, pp. 265-296, 1992.

[10] A. Ishimaru and R. L.-T. Cheung, "Multiple scattering effects on wave propagation due to rain," Ann. Telecommun., vol. 35, pp. 373-379, 1980.

[11] M. W. Whitt, "Microwave scattering from periodic row-structured vegetation," Ph.D. dissertation, Univ. Michigan, Ann Arbor, 1991.

[12] R. E. Kleinman and T. B. A. Senior, "Rayleigh scattering," in Low and High Frequency Asymptotics, V. K. Vardan and V. V. Varadan, Eds. Amsterdam, The Netherlands: North-Holland, 1986, ch. 1.

[13] J. R. Kendra, "Microwave remote sensing of snow: An empirical/theoretical scattering model for dense random media," Ph.D. dissertation, Univ. Michigan, Ann Arbor, 1995.

[14] L. Tsang, J. A. Kong, and R. T. Shin, Theory of Microwave Remote Sensing. New York: Wiley, 1985.

[15] M. A. Tassoudji, K. Sarabandi, and F. T. Ulaby, "Design consideration and implementation of the LCX polarimetric scatterometer (POLARSCAT)," Radiation Lab., Univ. Michigan, Ann Arbor, Tech. Rep. 022486-T-2, 1989.

[16] K. Sarabandi, F. T. Ulaby, and M. A. Tassoudji, "Calibration of polarimetric radar systems with good polarization isolation," IEEE Trans. Geosci. Remote Sensing, vol. 28, pp. 70-75, Jan. 1990.

[17] J. L. Zhou and A. L. Tits, "User's guide for FSQP version 3.3b: A Fortran code for solving constrained nonlinear (minimax) optimization problems, generating iterates satisfying all inequality and linear constraints," Syst. Res. Center, Univ. Maryland, College Park, Tech. Rep. TR-92-107r3, Sept. 1993.

[18] J. R. Kendra, F. T. Ulaby, and K. Sarabandi, "Snow probe for in situ determination of wetness and density," IEEE Trans. Geosci. Remote Sensing, vol. 32, pp. 1152-1159, Nov. 1994.

[19] F. T. Ulaby, R. K. Moore, and A. K. Fung, Microwave Remote Sensing, Active and Passive, vols. 1-3. Dedham, MA: Artech House, 1986.

[20] R. West, L. Tsang, and D. P. Winebrenner, "Dense medium radiative transfer theory for two scattering layers with a Rayleigh distribution of particle sizes," IEEE Trans. Geosci. Remote Sensing, vol. 31, pp. 426-437, Mar. 1993.

[21] K. Sarabandi, "Derivation of phase statistics from the Mueller matrix," Radio Sci., vol. 27, no. 5, pp. 553-560, 1992

[22] F. T. Ulaby, T. H. Bengal, M. C. Dobson, J. R. East, J. B. Garvin, and D. L. Evans, "Microwave dielectric properties of dry rocks," IEEE Trans. Geosci. Remote Sensing, vol. 28, pp. 325-336, May 1990.

[23] C. E. Mandt, "Microwave propagation and scattering in a dense distribution of spherical particles," M.S. thesis, Univ. Washington, Seattle, 1987.

[24] P. R. Siqueira and K. Sarabandi, "Method of moments evaluation of the two-dimensional quasicrystalline approximation," IEEE Trans. Antennas Propagat., vol. 44, pp. 1067-1077, Aug. 1998.

[25] Y. Kuga, F. T. Ulaby, T. F. Haddock, and R. D. DeRoo, "Millimeterwave radar scattering from snow: 1. radiative transfer model," Radio Sci., vol. 26, no. 2, pp. 329-341, 1991.

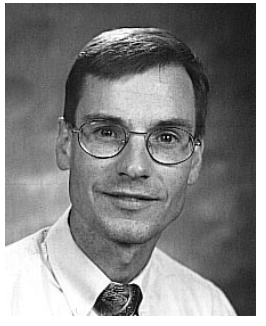

John R. Kendra (S'92-M'95) received the B.S.E.E. degree from the University of Houston, Houston, TX, in 1989 and the M.S.E.E. and $\mathrm{Ph} . D$. (electrical engineering) degrees from The University of Michigan, Ann Arbor, in 1990 and 1995, respectively. His dissertation dealt with experimental and theoretical studies of microwave scattering from dense random media, especially pertaining to snow. One aspect of this research was the development of an electromagnetic sensor, the Snow Probe, for in situ determination of snow

liquid water content.

$\mathrm{He}$ is currently with the $\mathrm{C}^{3} \mathrm{I}$ Group, Raytheon Systems Company, Dallas, TX, where he develops image processing algorithms for remote-sensing applications and algorithms pertaining to the calibration of multispectral and panchromatic sensors.

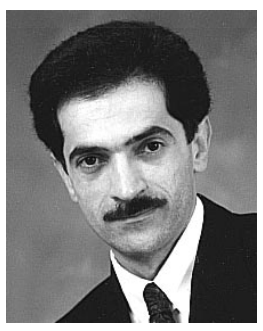

Kamal Sarabandi (S'87-M'90-SM'92) received the B.S. degree in electrical engineering from Sharif University of Technology, Tehran, Iran, in 1980 and the M.S.E. degree in electrical engineering in 1986, the M.S. degree in mathematics, and the Ph.D degree in electrical engineering in 1989, all from The University of Michigan, Ann Arbor.

He was a Microwave Engineer in the Telecommunication Research Center from 1980 to 1984 . He is currently an Associate Professor in the Department of Electrical Engineering and Computer Science, The University of Michigan. He has 15 years of experience with microwave sensors and radar systems. In the past eight years, he has served as the Principal Investigator and Co-Investigator on many projects sponsored by NASA, JPL, ARO, ONR, and GM, all related in one way or another to microwave and millimeter-wave radar remote sensing. He has published four book chapters and more than 80 papers in refereed journals on electromagnetic scattering, random media modeling, microwave measurement techniques, radar calibration, application of neural networks in inverse scattering problems, and microwave sensors. He has also had more than 130 papers and presentations in national and international conferences and symposia on similar subjects.

Dr. Sarabandi is listed in American Men and Women of Science and Who's Who in Electromagnetics. He has been a member of the IEEE Geoscience and Remote Sensing ADCOM since January 1998 and served as the Chairman of the Geoscience and Remote Sensing Society Southeastern Michigan chapter from 1991 to 1997 . He is also a member of Commission F of URSI and the Electromagnetic Academy. He was a recipient of a 1996 Teaching Excellence Award and the 1997 Henry Russel Award from the Regent of The University of Michigan. 